\title{
Time-varying total stiffness matrix of a rigid machine spindle-angular contact ball bearings assembly: Theory and analytical/experimental verifications
}

\author{
Fawzi M.A. El-Saeidy* \\ Faculty of Engineering and Information Technology, University of Technology, Sydney, Po Box 123, Broadway, \\ NSW 2007, Australia
}

Received 23 March 2009

Revised 22 March 2010

\begin{abstract}
A lagrangian formulation is presented for the total dynamic stiffness and damping matrices of a rigid rotor carrying noncentral rigid disk and supported on angular contact ball bearings (ACBBs). The bearing dynamic stiffness/damping marix is derived in terms of the bearing motions (displacements/rotations) and then the principal of virtual work is used to transfer it from the bearing location to the rotor mass center to obtain the total dynamic stiffness/damping matrix. The bearing analyses take into account the bearing nonlinearities, cage rotation and bearing axial preload. The coefficients of these time-dependent matrices are presented analytically. The equations of motion of a rigid rotor-ACBBs assembly are derived using Lagrange's equation. The proposed analyses on deriving the bearing stiffness matrix are verified against existing bearing analyses of SKF researchers that, in turn, were verified using both SKF softwares/experiments and we obtained typical agreements. The presented total stiffness matrix is applied to a typical grinding machine spindle studied experimentally by other researchers and excellent agreements are obtained between our analytical eigenvalues and the experimental ones. The effect of using the total full stiffness matrix versus using the total diagonal stiffness matrix on the natural frequencies and dynamic response of the rigid rotor-bearings system is studied. It is found that using the diagonal matrix affects natural frequencies values (except the axial frequency) and response amplitudes and pattern and causes important vibration tones to be missig from the response spectrum. Therefore it is recommended to use the full total stiffness matrix and not the diagonal matrix in the design/vibration analysis of these rotating machines. For a machine spindle-ACBBs assembly under mass unbalnce and a horizontal force at the spindle cutting nose when the bearing time-varying stiffness matrix (bearing cage rotation is considered) is used, the peak-to-valley variation in time domain of the stiffness matrix elements becomes significant compared to its counterpart when the bearing standard stiffness matrix (bearing cage rotation is neglected) is used. The vibration spectrum of the time-varying matrix case is marked by tones at bearing outer ring ball passing frequency, rotating unbalnce frequency and combination compared to spectrum of the standard stiffness matrix case which is marked by only the rotating unbalnce frequency. Therfore, it is highly recomended to model bearing stiffness matrix to be a time-dependent.
\end{abstract}

\section{Introduction}

Rotating rigid machinery incorporating rolling element bearings are used in many industrial applications. These machines are nonlinear and among sources of nonlinearities are the bearing nonlinear stiffness. Accurate calculation

\footnotetext{
${ }^{*}$ Corresponding author: Fawzi El-Saeidy, 58 Lowana Street, Villawood, NSW 2163, Australia. Tel./Fax: +612 97239181; E-mail: fawzi.elsaeidy@uts.edu.au.
} 
of the stiffness matrix of a rigid shaft supported on angular contact ball bearings is important in the stability analysis and eigenvalue problem solution of the system. The bearing model can be included in the machinery overall dynamic model by calculating the bearing dynamic loads vector or stiffness matrix and then assemble into the machinery overall load vector or stiffness matrix, respectively. Analytical studies that use five DOFs to calculate loads (forces/moments) of rolling element bearings supporting a rigid shaft are numerous [1-4]. There are many studies on calculating ball bearing stiffness matrix $\left(\mathbf{K}^{b}\right)$, see Section 1.1. The rotation of the bearing ball compliment (cage) around the bearing longitudnal axis makes $\mathbf{K}^{b}$ time-variant. To avoid complicated analyses different authors neglected effect of cage rotation and treated $\mathbf{K}^{b}$ to be time-invariant. $\mathbf{K}^{b}$ is computed using shaft motions at the bearing location (Section 2.4) and then used to derive the total stiffness matrix of the rigid shaft-bearings assembly $\left(\mathbf{K}^{c g}\right)$, see Section 2.5. To the author's best knowledge the studies related to $\mathbf{K}^{c g}$ are limited to [32-34], see Section 1.2.

\subsection{The rolling element bearing stiffness matrix}

Jones [5] presented a generalized theory to calculate rolling element bearings characteristics, namely,the displacements, contact loads (forces/moments) and stiffness matrix. He used a five-DOF (two lateral, one axial, two rotational) analysis and presented stiffness matrix coefficients analytically. The analyses are for an angular contact ball bearing (ACBB) and a radial roller bearing. Jones's work is adopted in numerous works [6-9]. De Mul et al. [6] used a five-DOF analysis for numeric calculations of ball bearings loads and stiffness matrix (no analytical expressions were presented for stiffness). In Ref. [7] a ball bearing $5 \times 5$ stiffness matrix coefficients are presented. The bearing is rigid except at the ball-to-races local hertzian contact, the ball centrifugal force and gyroscopic moment are neglected, and stiffness analysis is theoretical and similar to that of Jones [5] but using different vector notations. In Refs [5-7] bearing damping is neglected. Muhlner [8] used a five-DOF model and presented expressions of ACBB $5 \times 5$ stiffness matrix for the integrated analysis of bearings and structures. In Refs $[5,7,8]$, the necessary details of derivation of the stiffness matrix coefficients are not included and in the author's opinion this makes it difficult for the average reader to verify (check) these analyses before using them (see Section 3.1 of this study for corrections in Muhlner's [8] bearing stiffness matrix). Dietl [9] used a five-DOF ACBB and utilized principal of virtual work and derived an equation for numeric calculation of its stiffness matrix and added to it a geometrical stiffness (term) to account for the bearing axial preload. In Refs [5-9] the analyses are in fixed coordinates system and the stiffness matrix is time-invariant (i.e. effect of cage rotation is neglected). However, Ref. [8] presented plots of stiffness coefficients that show cyclic change due to the bearing cage rotation. Also Ref. [9] discussed cage rotation effect. The analyses of [8,9] are verified experimentally [9] and analytically using SKF software [8]. Shimizu and Tamura [24-26] used two-DOFs to calculate static stiffness of a deep groove ball bearing (DGBB) and showed that its variation is cyclic (due to change of the ball set position). El-Sayed [28] used his experimental results that show radial stiffness of a DGBB under only a radial dead load to increase nonlinearly as load increases and derived an emperical equation for stiffness calculation. El-Saeidy [30] showed that an increase in the rotating unbalance load of a rigid rotor-ideal ball bearings system will shift its frequencies other than forcing (unbalance) frequency to a higher frequency region; i.e. system's nonlinearity is of the hard spring type.

The works $[1-9,15,17-19,21-30,32-36,38-44,48,50-52]$ use summation over the bearing rolling elements in contact to compute bearing total loads. Houpert $[10,11]$ replaced this summation by integration using Sjovall's [12] axial and radial load distribution integrals $\left(J_{a}, J_{r}\right)$. Later Xernot et al. [13] used a five-DOF bearing anlaysis as [11] and presented coefficients of bearing stiffness matrix analytically. They used modified load integrals similar to $\left(J_{a}, J_{r}\right)$ in addition to a third integral. The three integrals need to be calculated numerically which in turn is an expensive process, and this makes Houpert's analysis of limited practical use, in the writer's opinion, compared to using summation over balls number. Houser et al. [14] used three-DOF (one radial, one axial, one rotational) bearing and presented equations for its radial, axial and moment loads using load distribution factors. They replaced integration needed to calculate these factors by summation over the load zone (not over balls number) and reported that their simple model can be used to compute a $3 \times 3$ stiffness matrix but no equation(s)/information on matrix computation were given. Sun et al. [15] presented equations of motion for dynamics of energy storage flywheel rigid system supported on active magnetic bearings and angular contact ball bearings. The supporting ACBB at each shaft end has three-DOFs (two lateral, one axial) for each of the inner and outer rings and the axial DOF is inertially and 
elastically uncoupled from the lateral DOFs. The ACBB lateral and axial stiffness coefficients only are included, as [6], (no report on effect of neglecting stiffness cross coupling coefficients), damping is taken to be constant, mass unbalance is included, and equations are solved using the Runge-Kutta method (RKM).

\subsection{Rigid shaft-angular contact ball bearings total stiffness matrix}

Jang and Jeong [32] used a five-DOF model to study effect of waviness of axially loaded ACBB on stability of a rigid rotor supported by two angular contact ball bearings. The mass centre of the rotor coincides with the bearings span centre. The three translational motions are taken to be uncoupled from each other and from the two rotational motions that in turn are coupled to each other only by gyroscopic effect. The authors neglected effect of bearing cage rotation, calculated stiffness matrix coefficients as [5], used diagonal stiffness matrix, and did not report on effect of neglecting the stiffness matrix off-diagonal coefficients. They presented time domain plots of the stiffness matrix coefficients that show that coefficients values are constant when the bearing is ideal (no waviness) and have cyclic variation when waviness is present. Liew and Lim [33] used a five-DOF bearing and presented without any information on its derivation a $5 \times 5$ stiffness matrix (denoted their by $[K(t)]$ ) of a rigid shaft of length $2 l_{r}$ carrying a central rigid disk and supported by one ACBB at each end. Applying our developed analyses of Eq. (54) to their rotor-bearings system shows that their $[K(t)]$ matrix needs corrections in its $[K]_{44},[K]_{45}$ and $[K]_{55}$ coefficients. In $[K]_{44}$, the part $\left\{\left[K_{b \theta_{x} y}\right]_{l p}+\left[K_{b \theta_{x} y}\right]_{r p}\right\} l_{r}$ reads as $2 l_{r}\left\{\left[K_{b \theta_{x} y}\right]_{l p}-\left[K_{b \theta_{x} y}\right]_{r p}\right\}$. In $[K]_{45}$, the part $\left\{\left[K_{b \theta_{x} x}\right]_{l p}+\left[K_{b \theta_{x} x}\right]_{r p}\right\} l_{r}$ reads as $l_{r}\left\{\left[K_{b \theta_{x} x}\right]_{l p}-\left[K_{b \theta_{x} x}\right]_{r p}\right\}$. In $[K]_{55}$, the part $\left\{\left[K_{b \theta_{y} x}\right]_{l p}+\left[K_{b \theta_{y} x}\right]_{r p}\right\} l_{r}$ reads as $2 l_{r}\left\{\left[K_{b \theta_{y} x}\right]_{l p}-\left[K_{b \theta_{y} x}\right]_{r p}\right\}$. Also, in the numerator of $K_{b z \theta_{y}}$ 's equation on page 1176 of Ref. [33], the term ' $+A_{s}^{2}$, should read ' $-A_{s}^{2}$, . Yahland [34] used a five-DOF model, neglected bearing cage rotation effect, used linearization approach and presented expressions of some coefficients (not all of them) of the linearized stiffness matrix of a rigid rotor supported on two angular contact ball bearings with waiveness.

\subsection{Rigid shaft-ball bearings system natural frequencies}

Analytical calculation of natural frequencies of a rigid shaft-angular contact ball bearings taking into account the bearing nonlinear stiffness is a complex task and this made different researchers use simple models to estimate frequencies [16-20]. Aini et al. [16] experimentally and analytically studied vibrations of a horizontal rigid grinding spindle-angular contact ball bearing system. They used a simple two-DOF (one lateral, one rotational) theoretical model, assumed the spindle bearings stiffness to be constant and calculated bounce and rock/pitch frequencies of the spindle assembly. The model does not account for axial mode frequency, ignores gyroscopic effect of the rotating shaft and requires preknowledge (i.e. linearization) of bearing stiffness. They compared the analytical results with experimental ones. Akturk [17,18] modeled a rigid shaft supported on two angular contact ball bearings using threeDOFs (two lateral, one axial). He linearized stiffness coefficient in the vertical direction around a constant deflection (= axial preload deflection) (no stiffness cross coupling coefficients). and presented an approximate equation for calculating vertical natural frequency. The equation is valid for axially preloaded bearing only (radial and other loads are not accounted for), requires linearized stiffness coefficient, ignores variation of the ball operating contact angle due to the bearing axial preload, and neglects gyroscopic effect of the rotating shaft. Vibration of axially loaded high speed rigid routing spindle supported on angular contact ball bearings runing under no cutting condition is analytically modeled using a three-DOF (one horizontal, one vertical, one axial) model and experimentally studied [19,20]. The spindle natural frequencies corresponding to the radial bounce mode and rocking mode are calculated by solving a simple frequency equation qutoted from Ref. [25] in Ref. [19] (the writer did not verify its derivation). The equation does not account for the axial mode frequency, assumes the stiffness coefficients of the supporting bearings to be linear (constant), and is applicable to stationary spindles only (gyroscopic effect of the rotating shaft is neglected).

Mevel and Guyader [21,22] used a two-DOF (one vertical, one horizontal) model to study vibrations of a DGBB under a vertical load. The load is assumed to be carried by one or two balls only at all times and the bearing horizontal and vertical natural frequencies are $\sqrt{k_{i i} / \text { mass }}, i=\mathrm{x}, \mathrm{y}$. The bearing horizontal and vertical stiffness coefficients $\left(k_{x x}, k_{y y}\right)$ are calculated as [23] ([23] used a two-DOF model and presented a method to calculate the $2 \times 2$ stiffness matrix of a radial ball bearing under vertical dead load). Yamamoto and Ishida [27] experimentally showed that shaft rotation causes cyclic variation of the bearing system natural frequency. While [29] used a simple one-DOF equation 
to calculate radial stiffness of a DGBB under radial load, calculated bearing radial natural frequency (bearing unit mass was used), and showed that frequency increases nonlinearly as load increases.

As can be seen the models on calculating natural frequencies of a rotor-rolling element system are for a simple two-DOF DGBB rotor and the analyses for a five-DOF ACBB rotor are incomplete and assume bearing stiffness to be constant (linear). In the writer's opinion stiffness linearization should be avoided as it is not an accurate estimation of the bearing stiffness coefficients and could cause a large difference between predicted and experimental results. For example in [31] experiments were conducted to measure radial stiffness of ball bearing under oscillating radial load and large difference between the measured stiffness $\left(12 \times 10^{8} \mathrm{~N} / \mathrm{m}\right)$ and calculated stiffness $\left(7 \times 10^{8} \mathrm{~N} / \mathrm{m}\right.$, it is not very clear how it was calculated) was reported which may be because the bearing was modeled using linear springs.

\subsection{The rolling element bearing damping}

The main sources of damping in a rolling element bearing are [9,35]: 1 . Material damping due to Hertzian deformation of the rolling element and raceways. 2. Damping due to sqeezing lubricant within entry region where oil is entrained into the Hertzian zone. 3. Elastohydrodynamic damping (EHD) lubrication film within the Hertzian contact zone. 4. Damping at inner ring-shaft interface and outer ring-housing interface. SKF experimental results show that sources 1 and 2 dominate the bearing damping. As rotor rotates, the stiff EHD layer develops and fixes the viscous damping mechanism within that zone and values of the damping coefficients converge to values of the dry (lubricant-free) bearing [35]. For dry deep grove ball bearings and dry angular contact ball bearings, the equivalent viscous damping coefficients are in the range 330-550 N.s/m [35]. However, equivalent viscous damping of constant $=200 \mathrm{~N} . \mathrm{s} / \mathrm{m}$ is used for deep groove ball bearing [36,50]. Further, different authors neglected bearing damping, see for example [1-4] and [30]. In Ref. [37] the damping coefficient at the ball-to-races contact is

$$
D=(0.25-2.5) 10^{-5} K \quad\left(\frac{N s}{\mu m}\right) .
$$

$K$ is the linearized hertzian stiffness constant $(N / \mu m)$ at the ball contact. Equation (1) is used to calculate ACBB damping matrix $(=$ constant $\times$ bearing stiffnes matrix $)$, see for example [32].

Cole et al. [38] modeled material damping force at a bearing ball-to-race contact $=\frac{3}{2} C^{b} K^{b} \dot{\delta} \delta^{n} . C^{b}=$ damping costant. $K^{b}=$ Hertz contact stiffness coefficient. $\delta=$ relative approach. $\dot{\delta}=$ relative approach velocity. In [8, 39] effect of lubricant in the bearing ball-to-race contacts is modeled using viscous linear damper of coefficient $C_{v}$ and the daming force of a ball in contact $=C_{v} \dot{\delta}$ for $\delta>0$. Without derivation details, Muhlner [8] presented analytical expressions for bearing $5 \times 5$ time-invariant damping matrix coefficients. He reported that $C_{v}$ can be determined analytically or experimentally as [9]. His analytical analyses on rotor-ball bearings produce results with good agreements with the experimental results of [9] and the analytical results obtained using the software used by SKF industries. Dietl [9] calculated the equivalent viscous material damping coefficient at the ball-to-race contact of a dry bearing $C_{v}=\psi \frac{K_{h}}{2 \pi f}$. $K_{h}$ is the bearing rolling element local Hertzian contact stiffness. $f$ is the vibrating bearing frequency. $\psi$ is a dimensionless loss factor. Then he calculated the dry bearing material damping matrix $\mathbf{C}_{B, h}$ using bearing stiffness matrix $\mathbf{K}_{B}$ according to

$$
\mathbf{C}_{B, h}=\psi \frac{\mathbf{K}_{B}}{2 \pi f} .
$$

In Refs [35,40], Eq. (2) is used with good agreement with experimental results.

As can be seen the studies on derivation of the $\mathbf{K}^{c g}$ matrix (Section 1.2) are very few and are incomplete and their applications are limited. None of these studies had reported on effect of using diagonal coefficients of $\mathbf{K}^{c g}$ marix versus full $\mathbf{K}^{c g}$ matrix. Moreover, these analyses, that use fixed coordinates system, present no details on the stiffness matrix derivation and this makes it difficult for the analyst to verify and/or extend them. Therefore the contributions of this study are:

(1) A Lagrangian formulation is presented for the time-dependent (cage rotation is considered) total stiffness matrix of a rigid rotor with noncentral disk supported on angular contact ball bearings. The coefficients of the total stiffness matrix are presented analytically. To the author's best knowlege the formulation is new. Also, for the first time, the derivation of the time-dependent bearing damping matrix is presented; 


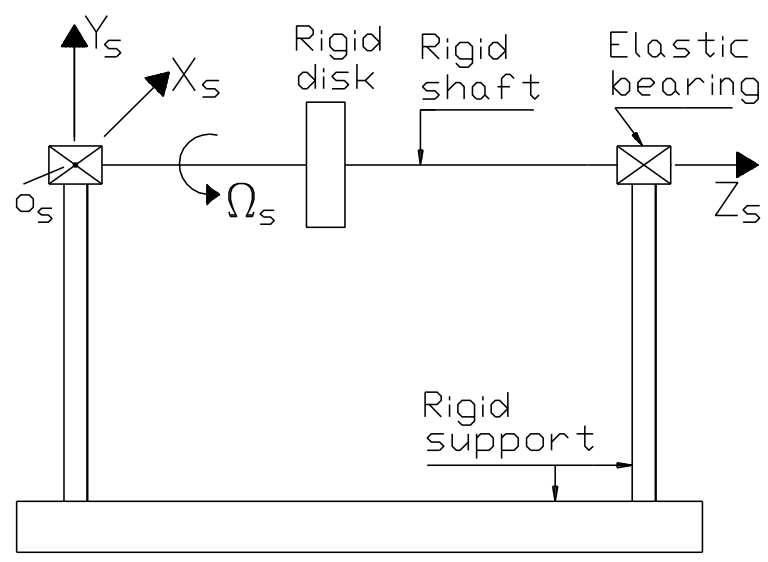

Fig. 1. A rigid rotor-elastic bearings-rigid support configuration.

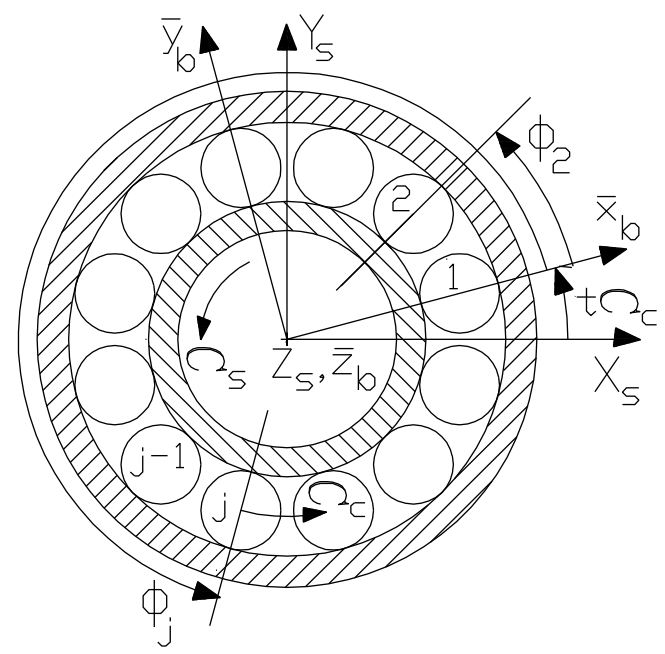

Fig. 2. Ball bearing elements and coordinate systems.

(2) Effect of using the total diagonal stiffness matrix $\left(\mathbf{K}_{\text {inc }}^{c g}\right)$ versus using the total full matrix $\left(\mathbf{K}^{c g}\right)$ on the rotor-bearings system natural frequencies and dynamics is investigated for the first time (Section 3.4.1);

(3) The formulation enables us to compute all eigenvalues (axial, bounce, rock) of the rotating/nonrotating rigid spindle-angular contact ball bearing assembly accurately without any need for linearization of the bearing stiffness (Section 3.3.2);

(4) Effect of using the time-dependent bearing stiffness matrix versus using the the standard formulation bearing stiffness matrix (i.e. cage rotation is neglected) is investigated (Section 3.4.2).

\section{Five-DOF rigid spindle-angular contact ball bearing stiffness matrix model}

Figure 1 shows a noncentral rigid disk mounted through its center to a rotating rigid shaft which in turn is mounted on two rolling element bearings. The bearings are mounted into their rigid housings that are firmly attached to a fixed rigid base (platform). The details of the bearing are depicted in Fig. 2. The position of the disk along shaft axis is at distance $L_{r}$ (not shown on Fig. 1) from the right bearing and at distance $L_{l}$ (not shown on Fig. 1) from the left bearing. The rigid shaft inertia is neglected compared to the disk inertia and therefore the system mass center is at the geometric center of the disk. The orientation of the vibrating rigid rotor in space (Fig. 3) is monitored using 


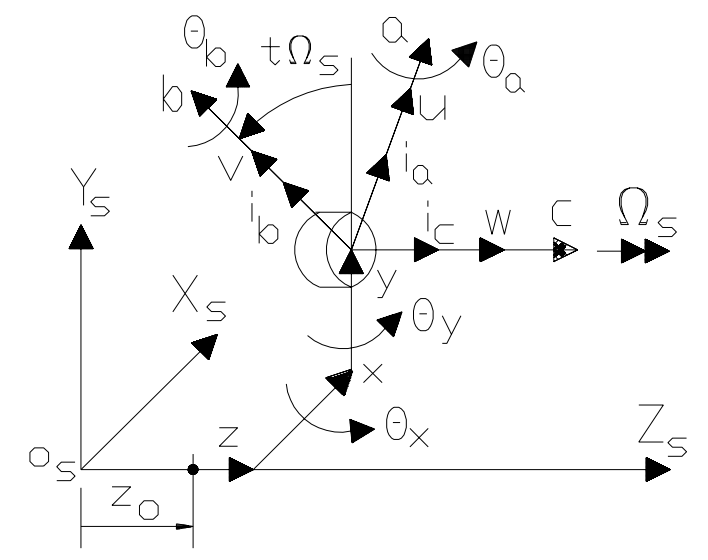

Fig. 3. Orientation of a rigid rotor in space.

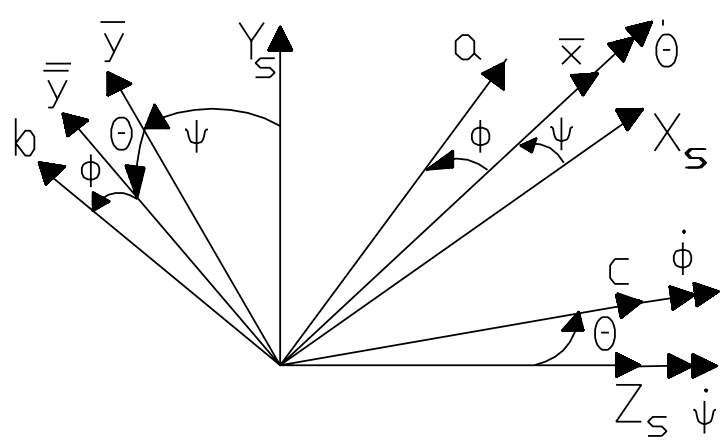

Fig. 4. Euler Angles.

Euler angles (Fig. 4). In Fig. 3, $X_{s} Y_{s} Z_{s}$ is an inertial frame (Fig. 1) and its origin (point $\mathrm{O}_{s}$ ) at the left bearing pedestal center. The triad $a b c$ is a body fixed coordinates system that rotates with the rotor differential element and represents its principal directions where $i_{a}, i_{b}$, and $i_{c}$ are unit vectors along axes $a, b$, and $c$, respectively. In Fig. 4 , $X_{s} Y_{s} Z_{s}$ is an inertial frame and $a b c$ is a body fixed one (see Fig. 3). $\bar{x} \bar{y} \bar{z}$ is an auxiliary, moving, frame initially coincides with the $X_{s} Y_{s} Z_{s}$. Euler angles are not unique and are adopted as the following:

1. Rotation $\psi$ about $Z_{s}$ axis results in $X_{s}$ coincides with $\bar{x}$;

2. Rotation $\theta$ about $\bar{x}$ results in the moving frame coincides with $c \bar{x} \bar{y}$;

3. Spin $\phi$ about $c$ axis results in the moving frame coincides with $a b c$ one.

The components of the rotor angular velocity vector $\bar{\omega}=\bar{\omega}_{a} i_{a}+\bar{\omega}_{b} i_{b}+\bar{\omega}_{c} i_{c}$ in the $a b c$ frame are [41].

$$
\left[\begin{array}{c}
\bar{\omega}_{a} \\
\bar{\omega}_{b} \\
\bar{\omega}_{c}
\end{array}\right]=\left[\begin{array}{c}
\dot{\psi} \sin \theta \sin \phi+\dot{\theta} \cos \phi \\
\dot{\psi} \sin \theta \cos \phi-\dot{\theta} \sin \phi \\
\dot{\psi} \cos \theta+\dot{\phi}
\end{array}\right] .
$$

$(\cdot)$ denotes differentiation with respect to time, $t$.

\subsection{The spindle mass center displacement vector and kinetic energy}

The disk mass center has five global DOFs: three translational along the $X_{s}, Y_{s}$ and $Z_{s}$, respectively, plus two rotational motions around the $X_{s}$ and $Y_{s}$ axes, respectively. The local displacement vector of the disk mass center is $\mathbf{h}=\left[\begin{array}{lllll}u & v & w & \theta_{a} & \theta_{b}\end{array}\right]^{T}$. Here $u, v$ and $w$ are the translational displacements along the $a, b$ and $c$ axes, respectively. $\theta_{a}$ and $\theta_{b}$ are the rotations around $a$ and $b$, respectively, where the superscript $T$ denotes a matrix 
transpose. The vector $\mathbf{h}$ is related to its counterpart global displacement vector $\mathbf{q}$ of the disk mass center relative to the machine base

$$
\mathbf{q}=\left[\begin{array}{lllll}
x & y & z & \theta_{x} & \theta_{y}
\end{array}\right]^{T} .
$$

measured in $\mathrm{X}_{s} \mathrm{Y}_{s} \mathrm{Z}_{s}$ system by transformation matrix $\overline{\mathbf{Q}}_{s}$ such that

$$
\begin{array}{ll}
\mathbf{h}=\overline{\mathbf{Q}}_{s} \mathbf{q}, & \overline{\mathbf{Q}}_{s}=\operatorname{diag}\left[\begin{array}{ll}
\mathbf{Q}_{t} & \mathbf{Q}_{r}
\end{array}\right], \\
\mathbf{Q}_{t}=\left[\begin{array}{ccc}
\cos \Omega_{s} t & \sin \Omega_{s} t & 0 \\
-\sin \Omega_{s} t & \cos \Omega_{s} t & 0 \\
0 & 0 & 1
\end{array}\right], \quad \mathbf{Q}_{r}=\left[\begin{array}{cc}
\cos \Omega_{s} t & \sin \Omega_{s} t \\
-\sin \Omega_{s} t & \cos \Omega_{s} t
\end{array}\right] .
\end{array}
$$

$\mathbf{Q}_{t}, \mathbf{Q}_{r}$ are orthogonal transformation matrices where subscripits $t$ and $r$ denote translational and rotational motions. In vector $\mathbf{q}$, the displacements $x, y$ and $z$ are along the $X_{s}, Y_{s}$ and $Z_{s}$ axes, respectively, and $\theta_{x}$ and $\theta_{y}$ are the rotational motions around the $X_{s}$ and $Y_{s}$ axes, respectively. The displacement vector q (Eq. (4)) is partitioned as

$$
\mathbf{q}=\left[\begin{array}{ll}
\mathbf{q}_{t}^{T} & \mathbf{q}_{r}^{T}
\end{array}\right]^{T}, \mathbf{q}_{t}=\left[\begin{array}{lll}
x & y & z
\end{array}\right]^{T}, \mathbf{q}_{r}=\left[\begin{array}{ll}
\theta_{x} & \theta_{y}
\end{array}\right]^{T} .
$$

The vectors $\mathbf{q}_{t}$ and $\mathbf{q}_{r}$ are related to vector $\mathbf{q}$ as

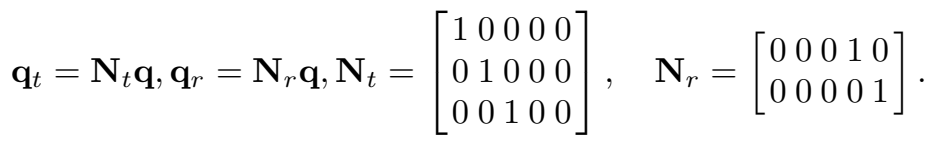

The absolute position vector of the disk mass center measured in the $X_{s} Y_{s} Z_{s}$ coordinates is $\mathbf{r}=\mathbf{r}_{o}+\mathbf{N}_{t} \mathbf{q}$. $\mathbf{r}_{o}=\left[\begin{array}{lll}x_{o} & y_{o} & z_{o}\end{array}\right]^{T}$ is the position vector of the mass center relative to the $X_{s} Y_{s} Z_{s}$ frame in the undisplaced state of the rigid shaft-elastic bearing system. The absolute translational velocity vector of the mass center is $\dot{\mathbf{r}}$.

$$
\dot{\mathbf{r}}=\mathbf{N}_{t} \dot{\mathbf{q}}, \quad \dot{\mathbf{q}}=\left[\begin{array}{lllll}
\dot{x} & \dot{y} & \dot{z} & \dot{\theta}_{x} & \dot{\theta}_{y}
\end{array}\right]^{T} .
$$

$\dot{\mathbf{q}}$ is the mass center generalized velocity vector. Expressed in the $X_{s} Y_{s} Z_{s}$ frame, the rotor translational kinetic energy $\mathcal{T}_{t}$ is

$$
\mathcal{T}_{t}=\frac{1}{2} \dot{\mathbf{r}}^{T} \mathbf{M}_{t} \dot{\mathbf{r}}, \quad \mathbf{M}_{t}=\operatorname{diag}\left[\begin{array}{lll}
m^{d} & m^{d} & m^{d}
\end{array}\right] .
$$

$\mathbf{M}_{t}$ is the system translational mass matrix and $m^{d}$ is the disk mass. $\dot{\mathbf{r}}$ is from Eq. (8). Expressed in the $a b c$ frame, the rotor rotational kinetic energy $\mathcal{T}_{r}$ is

$$
\mathcal{T}_{r}=\frac{1}{2} \mathbf{I}_{r}^{T} \mathbf{I}_{i} \mathbf{I}_{r}, \quad \mathbf{I}_{r}=\left[\begin{array}{lll}
\bar{\omega} \cdot i_{a} & \bar{\omega} \cdot i_{b} & \bar{\omega} \cdot i_{c}
\end{array}\right]^{T} .
$$

Vector $\bar{\omega}$ is from Eq. (3). (.) denotes a vector inner product. $\mathbf{I}_{i}=\operatorname{diag}\left[\begin{array}{lll}I_{d}^{d} & I_{d}^{d} & I_{p}^{d}\end{array}\right]$ is the rotor inertia tensor about $a, b$, and $c$ axes. $I_{p}^{d}$ is the polar moment of inertia of the rigid disk. $I_{d}^{d}$ is the disk diametral moment of inertia. From Fig. 4, we have for small angles of rotations $\theta_{x}=\theta \cos \psi$ and $\theta_{y}=\theta \sin \psi$. Differniate each of $\theta_{x}$ and $\theta_{y}$ with respect to time once, then

$$
\theta_{x}=\theta \cos \psi, \theta_{y}=\theta \sin \psi, \dot{\theta}_{x}=\dot{\theta} \cos \psi-\theta \dot{\psi} \sin \psi, \dot{\theta}_{y}=\dot{\theta} \sin \psi+\theta \dot{\psi} \cos \psi
$$

Using $\bar{\omega}_{c}$ from Eq. (3), we can show that

$$
\bar{\omega}_{c}^{2}=\Omega_{s}^{2}-\Omega_{s} \dot{\psi} \theta^{2} .
$$

where $\Omega_{s}=(\dot{\phi}+\dot{\psi})$ is the rotor spin speed. Using Eq. (11) one can show that $\dot{\theta}_{y} \theta_{x}-\dot{\theta}_{x} \theta_{y}=\dot{\psi} \theta^{2}$. Substitute this result into right hand side of Eq. (12) and use $\mathbf{q}_{r}=\left[\theta_{x}, \theta_{y}\right]^{T}$ (Eq. (6)) $=\mathbf{N}_{r} \mathbf{q}, \dot{\mathbf{q}}_{r}=\left[\dot{\theta}_{x}, \dot{\theta}_{y}\right]^{T}=\mathbf{N}_{r} \dot{\mathbf{q}}$, then

$$
\bar{\omega}_{c}^{2}=\Omega_{s}^{2}+\Omega_{s} \dot{\mathbf{q}}^{T} \mathbf{N}_{r}^{T} \widetilde{\mathbf{I}}_{22} \mathbf{N}_{r} \mathbf{q}, \quad \widetilde{\mathbf{I}}_{22}=\left[\begin{array}{cc}
0 & 1 \\
-1 & 0
\end{array}\right] .
$$

Using $\bar{\omega}_{b}$ and $\bar{\omega}_{c}$ from Eq. (3) and the approximation $\sin \theta \approx \theta$, then

$$
\bar{\omega}_{a}^{2}+\bar{\omega}_{b}^{2}=\dot{\theta}^{2}+\theta^{2} \dot{\psi}^{2} .
$$


Using Eq. (11), then $\dot{\theta}_{x}^{2}+\dot{\theta}_{y}^{2}=\dot{\theta}^{2}+\theta^{2} \dot{\psi}^{2}$. Substituting this result into Eq. (14) and recalling that $\dot{\mathbf{q}}_{r}=\mathbf{N}_{r} \dot{\mathbf{q}}$, we get

$$
\bar{\omega}_{a}^{2}+\bar{\omega}_{b}^{2}=\dot{\mathbf{q}}^{T} \mathbf{N}_{r}^{T} \mathbf{N}_{r} \dot{\mathbf{q}}
$$

Substitute from Eqs (13) and (15) into Eq. (10) to obtain $\mathcal{T}_{r}$ 's expression and substitute $\dot{\mathbf{r}}$ of Eq. (8) into $\mathcal{T}_{t}$ 's expression of Eq. (9) then the total kinetic energy $\mathcal{T}\left(=\mathcal{T}_{r}+\mathcal{T}_{t}\right)$ is given by.

$$
\mathcal{T}=\frac{1}{2}\left[\dot{\mathbf{q}}^{T} \overline{\mathbf{G}} \mathbf{q}+\dot{\mathbf{q}}^{T} \mathbf{M} \dot{\mathbf{q}}\right]
$$

where $\mathbf{M}$ is the disk inertia (mass) matrix. $\overline{\mathbf{G}}$ is its gyroscopic matrix.

$$
\mathbf{M}=\operatorname{diag}\left[\mathbf{M}_{t} \quad I_{d}^{d} \mathbf{I}_{22}\right], \quad \overline{\mathbf{G}}=\operatorname{diag}\left[\overline{\mathbf{0}}_{33} \quad \Omega_{s} I_{p}^{d} \widetilde{\mathbf{I}}_{22}\right] .
$$

Matrix $\mathbf{M}_{t}$ is from Eq. (9). $\mathbf{I}_{i i}$ is a $i \times i$ unit matrix. $\overline{\mathbf{0}}_{i j}$ is a $i \times j$ null matrix.

Let $\mu_{a}$ and $\mu_{b}$ be the disk mass center eccentricities in $a$ and $b$ directions. The disk generalized unbalance force vector is

$$
\mathbf{F}_{u}=\Omega_{s}^{2} \overline{\mathbf{Q}}_{s}^{T} \mathbf{M}\left[\begin{array}{lllll}
\mu_{a} & \mu_{b} & 0 & 0 & 0
\end{array}\right]^{T} .
$$

The matrix $\overline{\mathbf{Q}}_{s}$ is from Eq. (5) and matrix $\mathbf{M}$ is from Eq. (17).

\subsection{The bearing displacement vector}

Figure 2 depicts a ball bearing system where the global coordinate system $X_{s} Y_{s} Z_{s}$ has its origin at the bearing center with $Z_{s}$ axis coincides with the bearing axis. The frame $\bar{x}_{b} \bar{y}_{b} \bar{z}_{b}$ is a rotating coordinate system that spins at the bearing cage angular speed $\left(\Omega_{c} \mathrm{rad} \mathrm{s}^{-1}\right)$ where the $\bar{z}_{b}$ axis coincides with the bearing axis. The bearing inner ring is lightly fitted on its rigid shaft and is modeled as an integral part of it and thus rotates with the angular speed $\Omega_{s}$. The bearing outer ring, however, is fitted into its rigid and nonrotating housing. It should be noted that the velocities $\Omega_{s}$ and $\Omega_{c}$ are absolute. The bearing has five-DOFs: three oscillations along, respectively, the $X_{s}, Y_{s}$ and $Z_{s}$ axes plus two rotational motions around, respectively, the $X_{s}$ and $Y_{s}$ axes, The coupling between the bearing rotating elements and the rotating shaft is of the force-oscillations type. That is the input to bearing system is the shaft motions and in return, the generated bearing reaction loads are inputed to shaft at the bearing point of action. Let the global vibration amplitudes of the rotating shaft at the bearing point of action be $x^{b}, y^{b}$ and $z^{b}$, along $X_{s}, Y_{s}$ and $Z_{s}$ directions plus $\theta_{x}^{b}$ and $\theta_{y}^{b}$ around $X_{s}$ and $Y_{s}$ axes, respectively. These displacements, when transformed to the system $\bar{x}_{b} \bar{y}_{b} \bar{z}_{b}$ using the orthogonal transformation matrix $\overline{\mathbf{Q}}^{b}$ are given by $\bar{u}^{b}, \bar{u}^{b}, \bar{v}^{b}, \bar{\theta}_{u}^{b}$, and $\bar{\theta}_{v}^{b}$, respectively, such that

$$
\begin{aligned}
& \overline{\mathbf{u}}^{b}=\overline{\mathbf{Q}}^{b} \mathbf{q}^{b}, \quad \mathbf{q}^{b}=\mathbf{T}^{b} \mathbf{q}, \\
& \overline{\mathbf{u}}^{b}=\left[\begin{array}{lllllll}
\bar{u}^{b} & \bar{v}^{b} & \bar{w}^{b} & \bar{\theta}_{u}^{b} & \bar{\theta}_{v}^{b}
\end{array}\right]^{T}, \quad \mathbf{q}^{b}=\left[\begin{array}{lllll}
x^{b} & y^{b} & z^{b} & \theta_{x}^{b} & \theta_{y}^{b}
\end{array}\right]^{T} . \\
& \overline{\mathbf{Q}}^{b}=\operatorname{diag}\left[\begin{array}{ll}
\mathbf{Q}_{t}^{b} & \mathbf{Q}_{r}^{b}
\end{array}\right], \\
& \mathbf{Q}_{t}^{b}=\left[\begin{array}{ccc}
\cos \Omega_{c} t & \sin \Omega_{c} t & 0 \\
-\sin \Omega_{c} t & \cos \Omega_{c} t & 0 \\
0 & 0 & 1
\end{array}\right], \quad \mathbf{Q}_{r}^{b}=\left[\begin{array}{cc}
\cos \Omega_{c} t & \sin \Omega_{c} t \\
-\sin \Omega_{c} t & \cos \Omega_{c} t
\end{array}\right] .
\end{aligned}
$$

where the bearing geometric transformation matrix $\mathbf{T}^{b}$ is from Eq. (52) and $\mathbf{q}$ is from Eq. (4). For a rotating bearing inner and stationary outer ring, $\Omega_{c}=\frac{\Omega_{s}}{2}\left(1-\frac{D_{b} \cos \alpha_{o}}{2 r_{m}}\right) . r_{m}$ is the bearing balls set pitch radius. $D_{b}$ is the ball diameter. $\alpha_{o}$ is the ball unloaded contact angle. Differentiating vector $\overline{\mathbf{u}}^{b}$ of Eq. (19) with respect to time gives the bearing local velocity vector $\dot{\overline{\mathbf{u}}}^{b}$ such that

$$
\begin{aligned}
& \dot{\overline{\mathbf{u}}}^{b}=\overline{\mathbf{Q}}^{b} \dot{\mathbf{q}}^{b}+\dot{\overline{\mathbf{Q}}}^{b} \mathbf{q}^{b}, \quad \dot{\mathbf{q}}^{b}=\mathbf{T}^{b} \dot{\mathbf{q}}, \\
& \dot{\overline{\mathbf{u}}}^{b}=\left[\begin{array}{lllll}
\dot{\bar{u}}^{b} & \dot{\bar{v}}^{b} & \dot{\bar{w}}^{b} & \dot{\bar{\theta}}_{u}^{b} & \dot{\bar{\theta}}_{v}^{b}
\end{array}\right]^{T}, \dot{\mathbf{q}}^{b}=\left[\begin{array}{lllll}
\dot{x}^{b} & \dot{y}^{b} & \dot{z}^{b} & \dot{\theta}_{x}^{b} & \dot{\theta}_{y}^{b}
\end{array}\right]^{T} .
\end{aligned}
$$

where $\dot{\overline{\mathbf{Q}}}^{b}$ is the differentiation of the matrix $\overline{\mathbf{Q}}^{b}$ with respect to time. $\dot{\mathbf{q}}^{b}$ is the bearing global velocity vector and $\dot{\mathbf{q}}$ is from Eq. (8). The vector $\mathbf{q}^{b}$ which is given by Eq. (19) is obtained from the vector $\mathbf{q}$ of Eq. (6) of the mass center using the bearing geometric transformation matrix $\mathbf{T}^{b}$ of Eq. (52). 


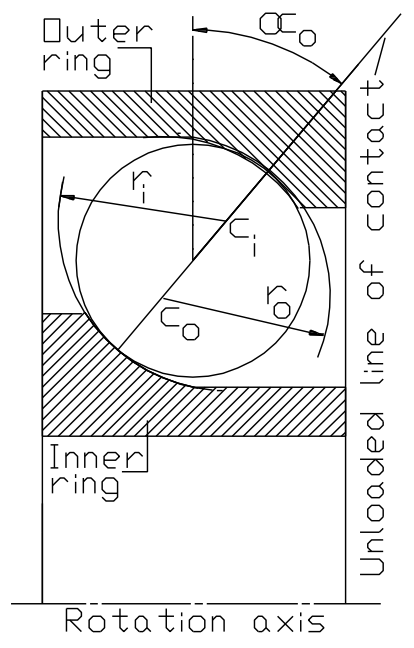

Fig. 5. Ball bearing ball-to-races contact configuration.

\subsection{The bearing elastic loads}

For the analysis of the bearing generated forces and moments, the outer raceway of groove curvature radius $r_{o}$ and center $c_{o}$ is taken to be fixed in the bearing housing and the inner raceway of groove curvature radius $r_{i}$ and center $c_{i}$ is attached to the shaft. If the bearing is unloaded, the relative distance between centers $c_{i}$ and $c_{o}$ along unloaded line of contact is $S_{o}=B D_{b} . B=\left(r_{o}+r_{i}-D_{b}\right) / D_{b}$ is the total curvature. see Fig. 5 which depicts the geometry of contact between the bearing rolling element (ball) and the bearing inner and outer races. The ball free contact angle $\alpha_{o}=\arccos \left(1-\frac{S_{d}}{2 S_{o}}\right) . S_{d}$ is the bearing diametral clearance. If the bearing is subjected to a pure axial load of magnitude $F_{a}^{b}$, the ensuing axial deformation $\bar{w}_{o}^{b}$ is

$$
\bar{w}_{o}^{b}=B D_{b} \frac{\sin \left(\alpha_{p}-\alpha_{o}\right)}{\cos \alpha_{p}} .
$$

The preload contact angle $\alpha_{p}$ is obtained by solving Eq. (23) iteratively.

$$
F_{a}^{b}=\bar{K}^{b} N b\left[B D_{b}\left(\frac{\cos \alpha_{o}}{\cos \alpha_{p}}-1\right)\right]^{n} \sin \alpha_{o} .
$$

$N b$ is the bearing rolling elements number. $\bar{K}^{b}$ is the Hertzian spring constant [42].

$$
\bar{K}^{b}=\left(\frac{1}{\left(1 / \bar{K}_{i}^{b}\right)^{1 / n}+\left(1 / \bar{K}_{o}^{b}\right)^{1 / n}}\right)^{n} .
$$

$\bar{K}_{i}^{b}$ and $\bar{K}_{o}^{b}$ are the contact stiffness constants at the inner and outer races. They are functions of the diameters of the bearing and balls, curvatures of the contact surfaces, the initial contact angles, and Hertzian stress. $n=1.5$ for ball bearings.

In the field, the supporting bearing could be loaded by a combination of axial, radial and moment loads/and or deformations. The relative distance between the centers $c_{i}$ and $c_{o}$ at the instantenous position of the $j t h$ ball is $S_{j}$.

$$
S_{j}=\sqrt{S_{r}^{2}+S_{a}^{2}}
$$

$S_{a}$ and $S_{r}$ are the axial and radial deformations at ball-to-races contact such that

$$
\begin{aligned}
& S_{a}=\bar{w}^{b}+\bar{w}_{o}^{b}+r_{m}\left(\bar{\theta}_{u}^{b} \sin \phi-\bar{\theta}_{v}^{b} \cos \phi\right)+S_{o} \sin \alpha_{o} . \\
& S_{r}=\bar{u}^{b} \cos \phi+\bar{v}^{b} \sin \phi+S_{o} \cos \alpha_{o} .
\end{aligned}
$$

The operating contact angle at the instantenous position of the $j t h$ ball is $\alpha_{j}$. 


$$
\tan \alpha_{j}=\frac{S_{a}}{S_{r}}, \quad \cos \alpha_{j}=\frac{S_{r}}{S_{j}}, \quad \sin \alpha_{j}=\frac{S_{a}}{S_{j}}
$$

If there is contact between ball and races, the ball elastic contact force $P_{j}^{b}$ is

$$
P_{j}^{b}=\bar{K}^{b}\left(S_{j}-S_{o}\right)^{n} \quad \text { if } \quad S_{j}>S_{o} .
$$

otherwise $P_{j}^{b}=0$. The bearing generated forces and moments when expressed in the $\bar{x}_{b} \bar{y}_{b} \bar{z}_{b}$ coordinates are given by the vector $\mathbf{f}_{e o}^{b}$ such that

$$
\mathbf{f}_{e o}^{b}=\left[\begin{array}{c}
f_{e x}^{b} \\
f_{e y}^{b} \\
f_{e z}^{b} \\
m_{e x}^{b} \\
m_{e y}^{b}
\end{array}\right]_{o}{ }_{j=1}^{N b} P_{j}^{b}\left[\begin{array}{c}
\cos \phi_{j} \cos \alpha_{j} \\
\sin \phi_{j} \cos \alpha_{j} \\
\sin \alpha_{j} \\
r_{m} \sin \phi_{j} \sin \alpha_{j} \\
-r_{m} \cos \phi_{j} \sin \alpha_{j}
\end{array}\right] .
$$

The subscript $e$ denotes bearing elastic spring loads (forces/moments). These load components when expressed in $X_{s} Y_{s} Z_{s}$ frame are given by load vector $\mathbf{F}_{e o}^{b}$.

$$
\mathbf{F}_{e o}^{b}=\left[\begin{array}{lllll}
F_{e x}^{b} & F_{e y}^{b} & F_{e z}^{b} & M_{e x}^{b} & M_{e y}^{b}
\end{array}\right]_{o}^{T}=\left(\overline{\mathbf{Q}}^{b}\right)^{T} \mathbf{f}_{e o}^{b} .
$$

Matrix $\overline{\mathbf{Q}}^{b}$ is from Eq. (20). The global load vector of the bearing inner ring, $\mathbf{F}_{e i}^{b}$, is

$$
\mathbf{F}_{e i}^{b}=-\mathbf{F}_{e o}^{b} \text {. }
$$

The outer subscripts $i$ and $o$ denote the bearing inner and outer races, respectively.

\subsection{The bearing stiffness matrix at bearing location, $\mathbf{K}^{b}$}

The coefficients $K_{i j}^{b}$ of the angular contact ball bearing stiffness matrix $\mathbf{K}^{b}$ are obtained by computing partial derivatives of the bearing load vector $\mathbf{F}_{e i}^{b}$ with respect to each displacement of the bearing displacement vector $\mathbf{q}^{b}$ of Eq. (19). From Eq. (31), the bearing force $F_{e x}^{b}$ is

$$
F_{e x}^{b}=f_{e x}^{b} \cos \Omega_{c} t-f_{e y}^{b} \sin \Omega_{c} t .
$$

The bearing local forces $f_{e x}^{b}$ and $f_{e y}^{b}$ are from Eq. (30). Thus,

$$
K_{11}^{b}=\frac{\partial F_{e x}^{b}}{\partial x^{b}}=\frac{\partial f_{e x}^{b}}{\partial x^{b}} \cos \Omega_{c} t-\frac{\partial f_{e y}^{b}}{\partial x^{b}} \sin \Omega_{c} t .
$$

Taking partial derivative of each of $f_{e x}^{b}$ and $f_{e y}^{b}$ with respect to displacement $x^{b}$, then

$$
\begin{aligned}
& \frac{\partial f_{e x}^{b}}{\partial x^{b}}=\sum_{j=1}^{N b}\left(\cos \alpha_{j} \frac{\partial P_{j}^{b}}{\partial x^{b}}+P_{j}^{b} \frac{\partial \cos \alpha_{j}}{\partial x^{b}}\right) \cos \phi_{j} . \\
& \frac{\partial f_{e y}^{b}}{\partial x^{b}}=\sum_{j=1}^{N b}\left(\cos \alpha_{j} \frac{\partial P_{j}^{b}}{\partial x^{b}}+P_{j}^{b} \frac{\partial \cos \alpha_{j}}{\partial x^{b}}\right) \sin \phi_{j} .
\end{aligned}
$$

Recalling from Eq. (29) that ball force $P_{j}^{b}=\bar{K}^{b}\left(S_{j}-S_{o}\right)^{n}$, then

$$
\frac{\partial P_{j}^{b}}{\partial x^{b}}=n \bar{K}^{b}\left(S_{j}-S_{o}\right)^{n-1} \frac{\partial S_{j}}{\partial x^{b}} .
$$

Taking partial derivative of $S_{j}$ of Eq. (25) with respect to $x^{b}$, then

$$
\frac{\partial S_{j}}{\partial x^{b}}=\frac{1}{S_{j}}\left(S_{r} \frac{\partial S_{r}}{\partial x^{b}}+S_{a} \frac{\partial S_{a}}{\partial x^{b}}\right) .
$$


Using Eqs (26) and (27), we obtain

$$
\begin{aligned}
& \frac{\partial S_{a}}{\partial x^{b}}=0 . \\
& \frac{\partial S_{r}}{\partial x^{b}}=\frac{\partial \bar{u}^{b}}{\partial x^{b}} \cos \phi_{j}+\frac{\partial \bar{v}^{b}}{\partial x^{b}} \sin \phi_{j} .
\end{aligned}
$$

From Eq. (19), we obtain

$$
\bar{u}^{b}=x^{b} \cos \Omega_{c} t+y^{b} \sin \Omega_{c} t, \quad \bar{v}^{b}=-x^{b} \sin \Omega_{c} t+y^{b} \cos \Omega_{c} t .
$$

Thus,

$$
\frac{\partial \bar{u}^{b}}{\partial x^{b}}=\cos \Omega_{c} t, \quad \frac{\partial \bar{v}^{b}}{\partial x^{b}}=-\sin \Omega_{c} t .
$$

Substituting from Eq. (42) into Eq. (40), then

$$
\frac{\partial S_{r}}{\partial x^{b}}=\cos \Omega_{c} t \cos \phi_{j}-\sin \Omega_{c} t \sin \phi_{j}=\cos \left(\Omega_{c} t+\phi_{j}\right) .
$$

Substitute from Eqs (39) and (43) into Eq. (38) and recall $\frac{S_{r}}{S_{j}}=\cos \alpha_{j}$ of Eq. (28), then

$$
\frac{\partial S_{j}}{\partial x^{b}}=\frac{S_{r}}{S_{j}} \cos \left(\Omega_{c} t+\phi_{j}\right)=\cos \alpha_{j} \cos \left(\Omega_{c} t+\phi_{j}\right) .
$$

Substitute from Eq. (44) into Eq. (37), then

$$
\frac{\partial P_{j}^{b}}{\partial x^{b}}=n \bar{K}^{b}\left(S_{j}-S_{o}\right)^{n-1} \cos \alpha_{j} \cos \left(\Omega_{c} t+\phi_{j}\right) .
$$

or

$$
\frac{\partial P_{j}^{b}}{\partial x^{b}}=\frac{n P_{j}^{b}}{S_{j}-S_{o}} \cos \alpha_{j} \cos \left(\Omega_{c} t+\phi_{j}\right) .
$$

Recalling from Eqs (28) that $\cos \alpha_{j}=S_{r} / S_{j}$, then

$$
\frac{\partial \cos \alpha_{j}}{\partial x^{b}}=\frac{S_{j} \frac{\partial S_{r}}{\partial x^{b}}-S_{r} \frac{\partial S_{j}}{\partial x^{b}}}{S_{j}^{2}} .
$$

Substituting from Eqs (43) and (44) into Eq. (47) and using the identity $\left(1-\cos ^{2} \alpha_{j}\right)=\sin ^{2} \alpha_{j}$, then

$$
\frac{\partial \cos \alpha_{j}}{\partial x^{b}}=\frac{\sin ^{2} \alpha_{j}}{S_{j}} \cos \left(\Omega_{c} t+\phi_{j}\right)
$$

Substituting from Eqs (46) and (48) into each of Eqs (35) and (36), then

$$
\begin{aligned}
& \frac{\partial f_{e x}^{b}}{\partial x^{b}}=\sum_{j=1}^{N b} P_{j}^{b}\left(\frac{n \cos ^{2} \alpha_{j}}{S_{j}-S_{o}}+\frac{\sin ^{2} \alpha_{j}}{S_{j}}\right) \cos \phi_{j} \cos \left(\Omega_{c} t+\phi_{j}\right) . \\
& \frac{\partial f_{e y}^{b}}{\partial x^{b}}=\sum_{j=1}^{N b} P_{j}^{b}\left(\frac{n \cos ^{2} \alpha_{j}}{S_{j}-S_{o}}+\frac{\sin ^{2} \alpha_{j}}{S_{j}}\right) \sin \phi_{j} \cos \left(\Omega_{c} t+\phi_{j}\right) .
\end{aligned}
$$

Substituting from Eqs (49) and (50) into Eq. (34), gives coefficient $K_{11}^{b}$ of $\mathbf{K}^{b}$

$$
K_{11}^{b}=\frac{\partial F_{e x}^{b}}{\partial x^{b}}=\sum_{j=1}^{N b} P_{j}^{b}\left(\frac{n \cos ^{2} \alpha_{j}}{S_{j}-S_{o}}+\frac{\sin ^{2} \alpha_{j}}{S_{j}}\right) \cos ^{2}\left(\Omega_{c} t+\phi_{j}\right) .
$$

Following the derivation of $K_{11}^{b}$, the rest of the stiffness matrix coefficients are derived accordingly. They are listed in Appendix A. 


\subsection{The bearing stiffness matrix at rotor mass center location, $\mathbf{K}^{c g}$}

Recall that disk is mounted at the distances $L_{r}$ and $L_{l}$ from the right and left bearings, respectively. Its displacement vector $\mathbf{q}$ measured in the $X_{s} Y_{s} Z_{s}$ frame is given by Eq. (4). For small oscillations and small angles of rotation, the right and left bearing displacement vectors, $\mathbf{q}_{r}^{b}=\left[\begin{array}{lllll}x_{r}^{b} & y_{r}^{b} & z_{r}^{b} & \theta_{x r}^{b} & \theta_{y r}^{b}\end{array}\right]^{T}$ and $\mathbf{q}_{l}^{b}=\left[\begin{array}{lllll}x_{l}^{b} & y_{l}^{b} & z_{l}^{b} & \theta_{x l}^{b} & \theta_{y l}^{b}\end{array}\right]^{T}$, respectively, measured in $X_{s} Y_{s} Z_{s}$ are

$$
\begin{array}{ccc}
\mathbf{q}_{r}^{b}= & \mathbf{T}_{r}^{b} \mathbf{q}, & \mathbf{q}_{l}^{b}=\mathbf{T}_{l}^{b} \mathbf{q} \\
\mathbf{T}_{r}^{b}=\left[\begin{array}{ccccc}
1 & 0 & 0 & 0 & L_{r} \\
0 & 1 & 0 & -L_{r} & 0 \\
0 & 0 & 1 & 0 & 0 \\
0 & 0 & 0 & 1 & 0 \\
0 & 0 & 0 & 0 & 1
\end{array}\right], & \mathbf{T}_{l}^{b}=\left[\begin{array}{ccccc}
1 & 0 & 0 & 0 & -L_{l} \\
0 & 1 & 0 & L_{l} & 0 \\
0 & 0 & 1 & 0 & 0 \\
0 & 0 & 0 & 1 & 0 \\
0 & 0 & 0 & 0 & 1
\end{array}\right]
\end{array}
$$

$\mathbf{T}_{r}^{b}$ and $\mathbf{T}_{l}^{b}$ are geometric transformation matrices for right and left bearings. Let $\mathbf{K}_{r}^{b}$ and $\mathbf{K}_{l}^{b}$ be the right and left bearing stiffness matrices, respectively, computed using Eqs (69) to (83) (Appendix A), where each matrix is computed at its bearing location, i.e. using the bearings displacement vectors $\mathbf{q}_{r}^{b}$ and $\mathbf{q}_{r}^{b}$, respectively, of Eq. (52). To transfer $\mathbf{K}_{r}^{b}$ and $\mathbf{K}_{l}^{b}$ to the mass center we use the principal of virtual work. The potential energy associated with the right bearing is $V_{r}^{b}=\frac{1}{2}\left(\mathbf{q}_{r}^{b}\right)^{T} \mathbf{K}_{r}^{b} \mathbf{q}_{r}^{b}$. Its first variation is $\delta V_{r}^{b}=\left(\mathbf{q}_{r}^{b}\right)^{T} \mathbf{K}_{r}^{b} \delta \mathbf{q}_{r}^{b}=\left(\delta \mathbf{q}_{r}^{b}\right)^{T} \mathbf{K}_{r}^{b} \mathbf{q}_{r}^{b} . \delta \mathbf{q}_{r}^{b}=\mathbf{T}_{r}^{b} \delta \mathbf{q}$ is the virtual change in vector $\mathbf{q}_{r}^{b}$ where $\delta \mathbf{q}$ is the virtual change in $\mathbf{q}$. Using $\mathbf{q}_{r}^{b}$ of Eq. (52), then $\delta V_{r}^{b}=(\delta \mathbf{q})^{T}\left(\mathbf{T}_{r}^{b}\right)^{T} \mathbf{K}_{r}^{b} \mathbf{T}_{r}^{b} \mathbf{q}$. The virtual work of the right bearing spring loads is $\delta W_{r}^{b}=-\delta V_{r}^{b}=-(\delta \mathbf{q})^{T}\left(\mathbf{T}_{r}^{b}\right)^{T} \mathbf{K}_{r}^{b} \mathbf{T}_{r}^{b} \mathbf{q}=-(\delta \mathbf{q})^{T} \mathbf{H}_{r}^{c g} \mathbf{q}$. Where $\mathbf{H}_{r}^{c g}=\left(\mathbf{T}_{r}^{b}\right)^{T} \mathbf{K}_{r}^{b} \mathbf{T}_{r}^{b}$ is the right bearing stiffness matrix expressed at the spindle-bearings assembly center of gravity. Likewise the left bearing stiffness matrix is $\mathbf{H}_{l}^{c g}=\left(\mathbf{T}_{l}^{b}\right)^{T} \mathbf{K}_{l}^{b} \mathbf{T}_{l}^{b}$. The rigid shaft-disk-elastic bearings system total stiffness matrix $\mathbf{K}^{c g}=\mathbf{H}_{r}^{c g}+\mathbf{H}_{l}^{c g}$ is

$$
\mathbf{K}^{c g}=\left(\mathbf{T}_{r}^{b}\right)^{T} \mathbf{K}_{r}^{b} \mathbf{T}_{r}^{b}+\left(\mathbf{T}_{l}^{b}\right)^{T} \mathbf{K}_{l}^{b} \mathbf{T}_{l}^{b}
$$

$\mathbf{K}^{c g}$ is time-varying due to rotation of the bearing cage. Let coefficients of matrices $\mathbf{K}_{r}^{b}, \mathbf{K}_{l}^{b}$ be $K_{i j}^{r}, K_{i j}^{l}, \mathrm{i}, \mathrm{j}=$ $1,2,3,4,5$, then coefficients of $\mathbf{K}^{c g}$ are $K_{i j}^{c g}$ such that

$$
\begin{aligned}
& K_{11}^{c g}=K_{11}^{r}+K_{11}^{l}, \quad K_{12}^{c g}=K_{12}^{r}+K_{12}^{l}, \quad K_{13}^{c g}=K_{13}^{r}+K_{13}^{l}, \\
& K_{14}^{c g}=K_{14}^{r}-L_{r} K_{12}^{r}+K_{14}^{l}+L_{l} K_{12}^{l}, \quad K_{15}^{c g}=K_{15}^{r}+L_{r} K_{11}^{r}+K_{15}^{l}-L_{l} K_{11}^{l}, \\
& K_{22}^{c g}=K_{22}^{r}+K_{22}^{l}, \quad K_{23}^{c g}=K_{23}^{r}+K_{23}^{l}, \quad K_{24}^{c g}=K_{24}^{r}-L_{r} K_{22}^{r}+K_{24}^{l}+L_{l} K_{22}^{l}, \\
& K_{25}^{c g}=K_{25}^{r}+L_{r} K_{21}^{r}+K_{25}^{l}-L_{l} K_{21}^{l}, \\
& K_{33}^{c g}=K_{33}^{r}+K_{33}^{l}, \quad K_{34}^{c g}=K_{34}^{r}-L_{r} K_{32}^{r}+K_{34}^{l}-L_{l} K_{32}^{l}, \\
& K_{35}^{c g}=K_{35}^{r}+L_{r} K_{31}^{r}+K_{35}^{l}-L_{l} K_{31}^{l}, \\
& K_{44}^{c g}=K_{44}^{r}-2 L_{r} K_{24}^{r}+L_{r}^{2} K_{22}^{r}+K_{44}^{l}+2 L_{l} K_{24}^{l}+L_{l}^{2} K_{22}^{l}, \\
& K_{45}^{c g}=K_{45}^{r}+L_{r}\left(K_{41}^{r}-K_{25}^{r}\right)-L_{r}^{2} K_{21}^{r}+K_{45}^{l}+L_{l}\left(K_{25}^{l}-K_{41}^{l}\right)-L_{l}^{2} K_{21}^{l}, \\
& K_{55}^{c g}=K_{55}^{r}+2 L_{r} K_{15}^{r}+L_{r}^{2} K_{11}^{r}+K_{55}^{l}-2 L_{l} K_{15}^{l}+L_{l}^{2} K_{11}^{l} .
\end{aligned}
$$

The above procedure is applicable to rotors supported on any number of bearings.

\subsection{The bearing damping matrix}

Damping in the rolling element bearing is modeled using viscous damper of constant coefficient $C_{v}^{b}$. If there is contact between the bearing ball and races, the ball local damping force $D_{j}^{b}$ is

$$
D_{j}^{b}=C_{v}^{b} \frac{d}{d t}\left(S_{j}-S_{o}\right)=C_{v}^{b} \dot{S}_{j}, \quad \text { if } \quad S_{j}>S_{o}
$$


otherwise, $D_{j}^{b}=0$. The generated damping forces/moments when expressed in the $\bar{x}_{b} \bar{y}_{b} \bar{z}_{b}$ frame are given by the vector $f_{d o}^{b}$ such that

$$
f_{d o}^{b}=\left[\begin{array}{c}
f_{d x}^{b} \\
f_{d y}^{b} \\
f_{d z}^{b} \\
m_{d x}^{b} \\
m_{d y}^{b}
\end{array}\right]_{o} \sum_{j=1}^{N b} D_{j}^{b}\left[\begin{array}{c}
\cos \phi_{j} \cos \alpha_{j} \\
\sin \phi_{j} \cos \alpha_{j} \\
\sin \alpha_{j} \\
r_{m} \sin \phi_{j} \sin \alpha_{j} \\
-r_{m} \cos \phi_{j} \sin \alpha_{j}
\end{array}\right] .
$$

Using $S_{j}$ Eq. (25), $\frac{S_{r}}{S_{j}}=\cos \alpha_{j}, \frac{S_{a}}{S_{j}}=\sin \alpha_{j}$ Eq. (28), then $D_{j}^{b}=C_{v}^{b} \dot{S}_{j}=C_{v}^{b}\left(\dot{S}_{r} \cos \alpha_{j}+\dot{S}_{a} \sin \alpha_{j}\right)$ which upon substituting into Eq. (56) gives

$$
\left[\begin{array}{c}
f_{d x}^{b} \\
f_{d y}^{b} \\
f_{d z}^{b} \\
m_{d x}^{b} \\
m_{d y}^{b}
\end{array}\right]_{o} \sum_{j=1}^{N b} C_{v}^{b}\left[\begin{array}{c}
\cos \phi_{j}\left(\dot{S}_{r} \cos ^{2} \alpha_{j}+\dot{S}_{a} \sin \alpha_{j} \cos \alpha_{j}\right) \\
\sin \phi_{\dot{j}}\left(\dot{S}_{r} \cos ^{2} \alpha_{j}+\dot{S}_{a} \sin \alpha_{j} \cos \alpha_{j}\right) \\
\left(\dot{S}_{r} \sin \alpha_{j} \cos \alpha_{j}+\dot{S}_{a} \sin ^{2} \alpha_{j}\right) \\
r_{m} \sin \phi_{j}\left(\dot{S}_{r} \sin \alpha_{j} \cos \alpha_{j}+\dot{S}_{a} \sin ^{2} \alpha_{j}\right) \\
-r_{m} \cos \phi_{j}\left(\dot{S}_{r} \sin \alpha_{j} \cos \alpha_{j}+\dot{S}_{a} \sin ^{2} \alpha_{j}\right)
\end{array}\right] .
$$

The inner subscript $d$ denotes damping and the other subscripts are defined before. The damping load vector $\mathbf{f}_{d o}^{b}$ when expressed in the $X_{s} Y_{s} Z_{s}$ frame is given by $\mathbf{F}_{d o}^{b}$.

$$
\mathbf{F}_{d o}^{b}=\left[\begin{array}{lllll}
F_{d x}^{b} & F_{d y}^{b} & F_{d z}^{b} & M_{d x}^{b} & M_{d y}^{b}
\end{array}\right]_{o}^{T}=\left(\overline{\mathbf{Q}}^{b}\right)^{T} \mathbf{f}_{d o}^{b} .
$$

The global damping load vector of the bearing inner ring is $\mathbf{F}_{d i}^{b}$, such that

$$
\mathbf{F}_{d i}^{b}=-\mathbf{F}_{d o}^{b} .
$$

The bearing damping matrix coefficients $\left(D_{i j}^{b}\right)$ are computed as follows. From Eq. (58), the bearing damping force $F_{d x}^{b}$ in the $X_{s}$ direction is

$$
F_{d x}^{b}=f_{d x}^{b} \cos \Omega_{c} t-f_{d y}^{b} \sin \Omega_{c} t .
$$

where the bearing local damping forces $f_{d x}^{b}$ and $f_{d y}^{b}$ are from Eq. (57). Thus, the coefficient $D_{11}^{b}$ of the bearing damping matrix $\mathbf{D}^{b}$ is

$$
D_{11}^{b}=\frac{\partial F_{d x}^{b}}{\partial \dot{x}^{b}}=\frac{\partial f_{d x}^{b}}{\partial \dot{x}^{b}} \cos \Omega_{c} t-\frac{\partial f_{d y}^{b}}{\partial \dot{x}^{b}} \sin \Omega_{c} t
$$

Take partial derivative of each of $f_{d x}^{b}$ and $f_{d y}^{b}$ with respect to velocity $\dot{x}^{b}$, then

$$
\left[\begin{array}{c}
\frac{\partial f_{d x}^{b}}{\partial \dot{x}^{b}} \\
\frac{\partial f_{d y}^{b}}{\partial \dot{x}^{b}}
\end{array}\right]=\sum_{j=1}^{N b} C_{v}^{b}\left[\begin{array}{l}
\cos \phi_{j}\left(\frac{\partial \dot{S}_{r}}{\partial \dot{x}^{b}} \cos ^{2} \alpha_{j}+\frac{\partial \dot{S}_{a}}{\partial \dot{x}^{b}} \sin \alpha_{j} \cos \alpha_{j}\right) \\
\sin \phi_{j}\left(\frac{\partial \dot{S}_{r}}{\partial \dot{x}^{b}} \cos ^{2} \alpha_{j}+\frac{\partial \dot{S}_{a}}{\partial \dot{x}^{b}} \sin \alpha_{j} \cos \alpha_{j}\right)
\end{array}\right] \text {. }
$$

Using Eq. (27), then $\dot{S}_{r}=\dot{\bar{u}}^{b} \cos \phi_{j}+\dot{\bar{v}}^{b} \sin \phi_{j}$. Thus $\frac{\partial \dot{S}_{r}}{\partial \dot{x}^{b}}=\frac{\partial \dot{u}^{b}}{\partial \dot{x}^{b}} \cos \phi_{j}+\frac{\partial \dot{v}^{b}}{\partial \dot{x}^{b}} \sin \phi_{j}$. But from Eqs (21), (19) and (20), we obtain

$$
\left[\begin{array}{c}
\dot{\bar{u}}^{b} \\
\dot{\bar{v}}^{b}
\end{array}\right]=\left[\begin{array}{c}
\dot{x}^{b} \cos \Omega_{c} t+\dot{y}^{b} \sin \Omega_{c} t+\Omega_{c}\left(-x^{b} \sin \Omega_{c} t+y^{b} \cos \Omega_{c} t\right) \\
-\dot{x}^{b} \sin \Omega_{c} t+\dot{y}^{b} \cos \Omega_{c} t-\Omega_{c}\left(x^{b} \cos \Omega_{c} t+y^{b} \sin \Omega_{c} t\right)
\end{array}\right],\left[\begin{array}{c}
\frac{\partial \dot{\bar{u}}^{b}}{\partial \dot{x}^{b}} \\
\frac{\partial \dot{v}^{b}}{\partial \dot{x}^{b}}
\end{array}\right]=\left[\begin{array}{c}
\cos \Omega_{c} t \\
-\sin \Omega_{c} t
\end{array}\right] .
$$

and therefore, $\frac{\partial \dot{S}_{r}}{\partial \dot{x}^{b}}=\cos \left(\Omega_{c} t+\phi_{j}\right)$ which upon substituting into Eq. (62) and since $\frac{\partial \dot{S}_{a}}{\partial \dot{x}^{b}}=0$, then

$$
\left[\begin{array}{c}
\frac{\partial f_{d x}^{b}}{\partial \dot{x}^{b}} \\
\frac{\partial f_{d y}^{b}}{\partial \dot{x}^{b}}
\end{array}\right]=\sum_{j=1}^{N b} C_{v}^{b}\left[\begin{array}{c}
\cos \phi_{j} \cos ^{2} \alpha_{j} \cos \left(\Omega_{c} t+\phi_{j}\right) \\
\sin \phi_{j} \cos ^{2} \alpha_{j} \cos \left(\Omega_{c} t+\phi_{j}\right)
\end{array}\right] \text {. }
$$


Table 1

ACBB 7039 parameters, Muhlner [8]

\begin{tabular}{llll}
\hline Item & value & Item & Value \\
\hline Pitch circle radius, $r_{m}$ & $36.25 \mathrm{~mm}$ & Ball diameter, $D_{b}$ & $17.7 \mathrm{~mm}$ \\
Outer race curvature radius, $r_{o}$ & $9.38 \mathrm{~mm}$ & Initial contact, $\alpha^{\circ}$ & $40^{\circ}$ \\
Inner race curvature radius, $r_{i}$ & $9.16 \mathrm{~mm}$ & Number of balls, $\mathrm{Nb}$ & 12 \\
\hline
\end{tabular}

Substituting from Eqs (64) into Eq. (61), then

$$
D_{11}^{b}=\sum_{j=1}^{N b} C_{v}^{b} \cos ^{2} \alpha_{j} \cos ^{2}\left(\Omega_{c} t+\phi_{j}\right) .
$$

Following derivation of $D_{11}^{b}$, the rest of damping matrix coefficients are derived accordingly and listed in Appendix B. Now, total damping matrix of the rigid shaft-disk-elastic bearings system, expressed ( acting) at mass centre is $\mathbf{D}^{c g}$.

$$
\mathbf{D}^{c g}=\left(\mathbf{T}_{r}^{b}\right)^{T} \mathbf{D}_{r}^{b} \mathbf{T}_{r}^{b}+\left(\mathbf{T}_{l}^{b}\right)^{T} \mathbf{D}_{l}^{b} \mathbf{T}_{l}^{b} .
$$

$\mathbf{D}_{r}^{b}$ and $\mathbf{D}_{l}^{b}$ are damping matrices of the right and left bearings, respectively, each is derived at its bearing position. The matrices $\mathbf{T}_{r}^{b}$ and $\mathbf{T}_{l}^{b}$ are from Eq. (52).

\subsection{Equations of motion}

The potential and dissipation functions of the rotor-rolling element bearings system are $\mathcal{P}=\frac{1}{2} \mathbf{q}^{T} \mathbf{K}^{c g} \mathbf{q}$ and $\mathcal{D}=\frac{1}{2} \dot{\mathbf{q}}^{T} \mathbf{D}^{c g} \dot{\mathbf{q}}$, respectively. The stiffness matrix $\mathbf{K}^{c g}$ is from Eq. (53) and displacement vector $\mathbf{q}$ is from (4). The dampig matrix is from Eq. (66) and velocity vector $\dot{\mathbf{q}}$ is from Eq. (8). Recall $\mathcal{T}$ of Eq. (16) and $\mathbf{F}_{u}$ of Eq. (18). Apply Lagrange's equations $\frac{d}{d t}\left(\frac{\partial \mathcal{T}}{\partial \dot{\mathbf{q}}_{k}}\right)-\frac{\partial \mathcal{T}}{\partial \mathbf{q}_{k}}+\frac{\partial \mathcal{P}}{\partial \mathbf{q}_{k}}+\frac{\partial \mathcal{D}}{\partial \dot{\mathbf{q}}_{k}}=\mathbf{F}_{u k}, \quad k=x, y, z, \theta_{x}, \theta_{y}$, we obtain

$$
\mathbf{M} \ddot{\mathbf{q}}+\mathbf{D}^{c g} \dot{\mathbf{q}}+\mathbf{G} \dot{\mathbf{q}}+\mathbf{K}^{c g} \mathbf{q}=\mathbf{F}, \quad \mathbf{G}=\frac{1}{2}\left[\overline{\mathbf{G}}-\overline{\mathbf{G}}^{T}\right], \quad \mathbf{F}=\mathbf{F}_{u} .
$$

$\mathbf{G}$ is the gyroscopic matrix where the matrix $\overline{\mathbf{G}}$ is from Eq. (17). $\mathbf{F}$ is the load vector.

\section{The bearing model verifications/application}

\subsection{Bearing stiffness matrix-comparison with SKF results/software}

The angular contact ball bearing (ACBB) 7039 whose data are given in Table 1 is subjected to external load vector $\left(\mathbf{F}_{e x t}^{b}\right)$ of a radial force of $500 \mathrm{~N}$ in the horizontal direction, a radial force of $500 \mathrm{~N}$ in the vertical direction, an axial force of $2000 \mathrm{~N}$, a moment of 1.0 N.m about horizontal axis, and a moment of 1.0 N.m about vertical axis (Muhlner [8]). As a result, the bearing system will experience an unknown deformation vector $\mathbf{q}^{b}$ needed to maintain the static equilibrium. The deformations $\mathbf{q}^{b}$ are determined using Newton-Raphson method for a system of simultaneous nonlinear equations (details are not included for brevity). Then vector $\mathbf{q}^{b}$ is used to calculate bearing loads and stiffness matrix $\mathbf{K}^{b}$ using the analytical analyses developed above. We observe that Muhlner's stiffness matrix coefficients $\left(\Delta K_{H i, j_{q}}\right)$ listed on pages 113-114 in [8] require corrections, e.g. $\Delta K_{H_{5,5 q}}=$ $H_{5} r_{m}^{2}\left(1.5 H_{1} \sin \beta-H_{4}^{2} H_{2}^{2}\right) \sin ^{\varphi}$ should read $\Delta K_{H_{5,5}}=H_{5} r_{m}^{2}\left(1.5 H_{1} \sin \beta-H_{4}^{2} H_{2}^{2}\right) \sin ^{2} \varphi$. Also, in Eq. (A.22) of [9] (which is a part of Dietl's stiffness matrix), the term $\left(r \cos \alpha_{i} \cos \varphi_{i}\right)$ should read as $\left(r \cos \alpha_{i} \sin \varphi_{i}\right)$. The coefficients of the bearing stiffness matrix are listed in Table 2 for different models. Our proposed model produces excellent results compared to both Muhlner's model [8] (the maximum absolute error is $0.81 \%$ ) and Dietl's model [9] (the maximum absolute error is $2.55 \%$ ) that in turn were validated against both SKF software (not available for public engineers) and experimental results. Recall that Dietl's bearing stiffness matrix has two parts; namely, one part called linear-elastic bearing stiffness matrix and one part called geometrical (preload) stiffness matrix and part due to preload (geometrical stiffness matrix) is approximate. 
Table 2

ACBB 7039 Stiffness matrix coefficients, $K_{i j}^{b}$, computed by different models. External load

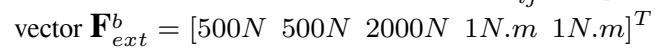

\begin{tabular}{|c|c|c|c|c|c|}
\hline$K_{i j}^{b}$ & Muhlner $^{b}[8]$ & $\% e r r^{a b}$ & DAMRO- $1^{\mathrm{a}}$ & Dietl $^{\mathrm{c}}$ [9] & $\% e r r^{\mathrm{ac}}$ \\
\hline $\mathrm{K}_{11}^{b}, \mathrm{~N} \mathrm{~mm}^{-1}$ & 171000.0 & -0.66 & 172131.6 & 172131.6 & 0.0 \\
\hline $\mathrm{K}_{12}^{b}, \mathrm{~N} \mathrm{~mm}^{-1}$ & 53300.0 & -0.75 & 53698.3 & 53698.3 & 0.0 \\
\hline $\mathrm{K}_{13}^{b}, \mathrm{~N} \mathrm{~mm}^{-1}$ & -4300.0 & 0.38 & -4283.6 & -4283.6 & 0.0 \\
\hline $\mathrm{K}_{14}^{b}, \mathrm{~N} \mathrm{rad}^{-1}$ & 1460000.0 & -0.81 & 1471843.7 & 1474665.0 & 0.19 \\
\hline $\mathrm{K}_{15}^{b}, \mathrm{~N} \mathrm{rad}^{-1}$ & -4880000.0 & -0.48 & -4903232.7 & -4903232.7 & 0.0 \\
\hline $\mathrm{K}_{22}^{b}, \mathrm{~N} \mathrm{~mm}^{-1}$ & 155000.0 & -0.34 & 155520.7 & 155520.7 & 0.0 \\
\hline $\mathrm{K}_{23}^{b}, \mathrm{~N} \mathrm{~mm}^{-1}$ & 11400.0 & 0.008 & 11399.1 & 11399.1 & 0.0 \\
\hline $\mathrm{K}_{24}^{b}, \mathrm{~N} \mathrm{rad}^{-1}$ & 4390000.0 & -0.45 & 4409792.6 & 4414719.7 & 0.11 \\
\hline $\mathrm{K}_{25}^{b}, \mathrm{~N} \mathrm{rad}^{-1}$ & -1500000.0 & -0.62 & -1509343.5 & -1471843.7 & -2.55 \\
\hline $\mathrm{K}_{33}^{b}, \mathrm{~N} \mathrm{~mm}^{-1}$ & 225000.0 & -0.45 & 226011.1 & 226011.1 & 0.0 \\
\hline $\mathrm{K}_{34}^{b}, \mathrm{~N} \mathrm{rad}^{-1}$ & -863000.0 & -0.68 & -868886.0 & -885433.0 & 1.87 \\
\hline $\mathrm{K}_{35}^{b}, \mathrm{~N} \mathrm{rad}^{-1}$ & 1550000.0 & -0.03 & 1550500.8 & 1550500.8 & 0.0 \\
\hline $\mathrm{K}_{44}^{b}, \mathrm{~N} \mathrm{~mm} / \mathrm{rad}$ & 139300000.0 & -0.003 & 139304365.4 & 139304365.4 & 0.0 \\
\hline $\mathrm{K}_{45}^{b}, \mathrm{~N} \mathrm{~mm} / \mathrm{rad}$ & -49400000.0 & -0.79 & -49788394.5 & -49563464.7 & -0.45 \\
\hline $\mathrm{K}_{55}^{b}, \mathrm{~N} \mathrm{~mm} / \mathrm{rad}$ & 157000000.0 & -0.10 & 157160958.5 & 157160958.5 & 0.0 \\
\hline
\end{tabular}

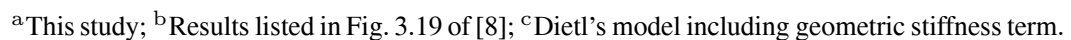
$\% \operatorname{err}^{\mathrm{ab}}=\frac{\text { Muhlner }[8]-(D A M R O-1)}{\text { Muhlner }[8]} \times 100, \% \operatorname{err}^{\mathrm{ac}}=\frac{\operatorname{Dietl}[9]-(D A M R O-1)}{\operatorname{Dietl}[9]} \times 100$

Table 3

The bearing Parameters, [44]

\begin{tabular}{lccc}
\hline Item & value & Item & Value \\
\hline Inner raceway radius, $R_{i}$ & $26.145 \mathrm{~mm}$ & shaft speed, $\Omega_{s}$ & $400 \mathrm{rad} / \mathrm{s}$ \\
Outer raceway radius, $R_{o}$ & $38.855 \mathrm{~mm}$ & Ball diameter, $D_{b}$ & $12.7 \mathrm{~mm}$ \\
Outer race curvature radius, $r_{o}$ & $6.6 \mathrm{~mm}$ & Number of balls, $N b$ & 9 \\
Inner race curvature radius, $r_{i}$ & $6.6 \mathrm{~mm}$ & & \\
\hline
\end{tabular}

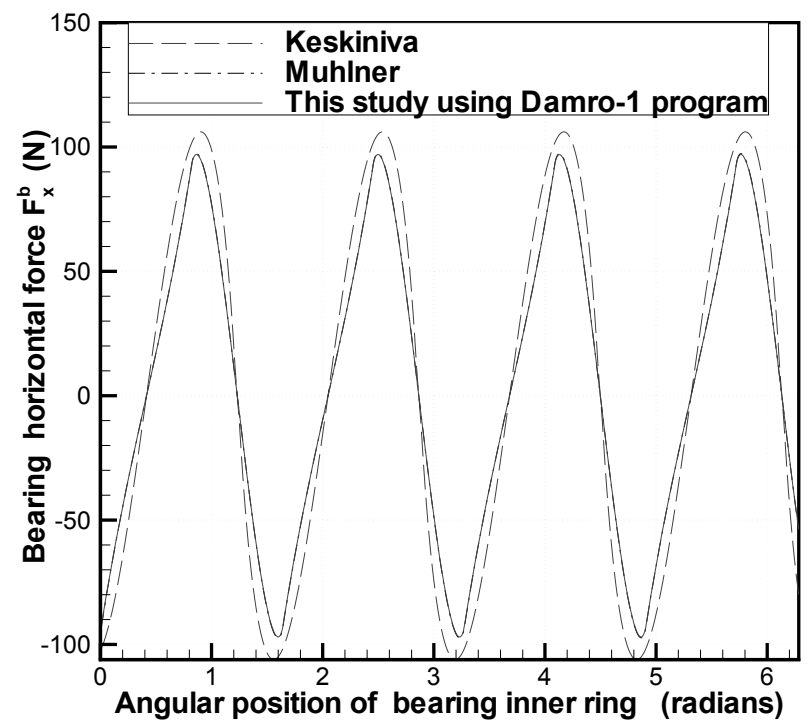

Fig. 6. Bearing horizontal force $F_{x}^{b}$ vs shaft rotation for $\Omega_{s}=400 \mathrm{rad} / \mathrm{s}$ and vertical displacement $e_{y}=-0.061 \mathrm{~mm}$; This study (solid line); Kiskiniva's model [44] (dashed line); Muhlner's model [8] (dash-dot line).

\subsection{Rotating bearing generatd dynamic loads due to external constant displacement}

The variable elastic compliance induced vibrations occur in a bearing under constant radial load or combined load but not under pure axial load [49]. The bearing example presented in [44] is studied. The bearing whose data are 
Table 4

The Rotor-SKF6002 bearing Parameters, [50]

\begin{tabular}{lccc}
\hline Item & value & Item & Value \\
\hline Inner raceway radius, $R_{i}$ & $9.37 \mathrm{~mm}$ & Shaft speed, $\Omega_{s}$ & $2000 \mathrm{rpm}$ \\
Outer raceway radius, $R_{o}$ & $14.13 \mathrm{~mm}$ & Cage speed, $\Omega_{c}$ & $=\frac{\Omega_{s} R_{i}}{\left(R_{i}+R_{o}\right)}$ \\
Radial clearance & $3.0 \mu \mathrm{m}$ & Balls number, $N b$ & 9 \\
Spring constant, $\bar{K}^{b}$ & $7.055 \times 10^{9} \mathrm{~N} \cdot \mathrm{m}^{-1.5}$ & Weight, $W$ & $6 \mathrm{~N}$ \\
Bearing damping, $C_{v}^{b}$ & $200 \mathrm{~N} . \mathrm{s} / \mathrm{m}$ & Unbalance force, $\mathrm{F}_{u}$ & $0.15 W$ \\
\hline
\end{tabular}

Table 5

Rotor vertical displacement spectra of this study and Ref. [50]

\begin{tabular}{lll}
\hline Freq. $(\mathrm{Hz})$ & Fig. 7 of this study & Fig. 19 of Ref. [50] \\
\hline $\mathrm{f}_{o}-3 f_{s}$ & $\sqrt{ }$ & not visible \\
$\mathrm{f}_{s}=33.333$ & $\sqrt{ }$ (dominant;amp=0.035 $\mu \mathrm{m})$ & $\sqrt{ }$ (dominant;amp $\approx 0.0325 \mu \mathrm{m})$ \\
$\mathrm{f}_{o}-2 f_{s}$ & $\sqrt{ }$ & $\sqrt{ }$ \\
$2 \mathrm{f}_{s}$ & $\sqrt{ }$ & $\sqrt{ }$ \\
$\mathrm{f}_{o}-f_{s}$ & $\sqrt{ }$ & $\sqrt{ }$ \\
$3 \mathrm{f}_{s}$ & $\sqrt{ }$ & $\sqrt{ }$ \\
$\mathrm{f}_{o}=119.617$ & $\sqrt{ }$ & $\sqrt{ }$ \\
$\mathrm{f}_{o}+f_{s}$ & $\sqrt{ }$ & $\sqrt{ }$ \\
$\mathrm{f}_{o}+2 f_{s}$ & $\sqrt{ }$ & $\sqrt{ }$ \\
\hline
\end{tabular}

given in Table 3 is given a constant vertical displacement $e_{y}=-0.061 \mathrm{~mm}$ and the bearing forces are computed while the shaft makes one revolution.

Kiskiniva [44] presented equations for calculating forces and moments acting on ACBB inner ring. He calculated the bearing Hertzian contact stiffness coefficient using the approximate method of [45-47] which requires calculation of the contacting bodies radii of curvature that in turn depend on the ball contact angle. But he assumed ball contact angle to be of zero value. However, this assumption of zero value introduces small error [48]. The bearing forces are calculated using our analyses of Eqs (19) to (32). Analysis of [44] is used to reproduce the plot of the bearing horizontal force $F_{x}^{b}$ against shaft rotation of Fig. 3.12 in [44]. The plot is shown in Fig. 6 (dashed line) along with our model plot (solid line) and Muhlner's model plot (dash-dot line). Our model and Muhlner's model produce typical results. The small difference between our results and Kiskiniva results may be due to approximation stated above. However, there is good qualitative and quantitative agreement. Variation in $F_{x}^{b}$ is periodic of period $\approx 0.00433 \mathrm{~s}$. i.e. $230.947 \mathrm{~Hz}$ which could be due to $f_{o}=\mathrm{Nb} f_{c}=230.625 \mathrm{~Hz}$ (the outer ring ball passing frequency, also known as elastic variable compliance frequency), $f_{c}=25.625 \mathrm{~Hz}$ is the bearing cage frequency. i.e. variation is due to the bearing variable elastic compliance. Moreover, the variation is not pure sinsoidal as harmonic distortion (due to bearing nonlinearity) is visible. These observations agree with similar SKF analytical/experimental observations on response of ball bearings under pure constant radial loads [49].

\subsection{Comparison with experimental/analytical results}

\subsubsection{Vibration of unbalanced two-DOF rigid rotor-ball bearings}

Tiwari et al. [50] used two-DOF (1 horizontal, 1 vertical) model and analytically and experimentally studied effect of SKF6002 deep grrove ball bearing internal clearance on dynamics of a horizontal unbalanced rigid rotor under constant vertical load. The bearing horizontal and vertical nonlinear forces are caculated taking into account cage rotation and radial clearance. They solved their two second order equations of motion using RKM and validated numerical results using experimental test rig which is described in Fig. 21 in [50]. The rotor-bearing parameters are listed in Table 4. We applied our developed analyses to this rotor-bearing system and obtained response using RKM. Time domain record is zero meaned and windowed using Hanning window and FFT is computed using DAMRO-1 program [43]. Figure 7 depicts FFT of the bearing vertical displacement where we see excellent qualitative and quantitative agreement with FFT of the vertical motion given in Eq. (19) in [50]. In each of our FFT and FFT of [50], the dominant vibration is at $f_{s}$ (the rotor rotational speed frequency) and its amplitude is about $0.035 \mu \mathrm{m}$. The comparison between our result and that of [50] is listed in Table 5. $f_{o}$ is the variable elastic compliance frequency (defined above) and $f_{s}$ is the unbalance frequency. In [50], $V C$ and $n X, n=1,2,3$, correspond to $f_{o}$ and $n f_{s}$, 


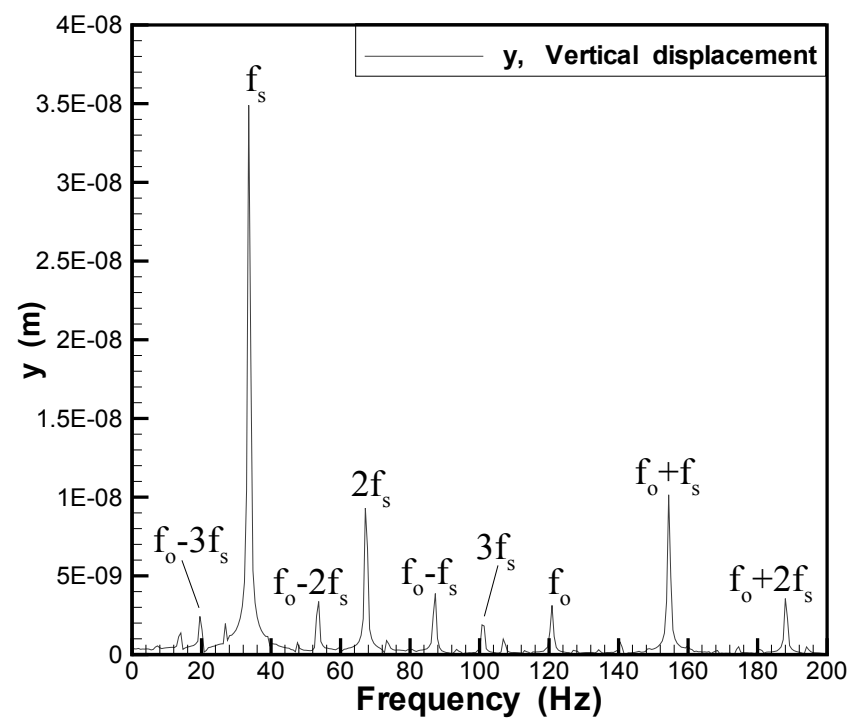

Fig. 7. FFT of the mass center (C.G) vertical displacement of deep groove ball bearing (DGBB) SKF6002.

respectively. FFT is marked with vibration tones at $f_{o}, f_{s}$ and its higher harmonics in addition to peaks at $\left(f_{o} \pm n f_{s}\right)$ and this agrees with the analytical and experimental results of Ref. [50].

3.3.2. Natural frequencies of five-DOF experimental rigid grinding spindle-angular contact ball bearings assembly

The experimental grinding spindle depicted in Fig. 1 of [16] and 4 of [51] is analytically simulated. The shaft is mounted on a pair of angular contact ball bearings (RHP 7008). "The bearings are slide and interference fitted into the housing and on the shaft". "Hence a nearly clearance-free radial fit is ensured" [16]. The bearings-spindle parameters are listed in Table 6. Using these parameters, the rigid spindle-elastic bearings symmetric total stiffness matrix is calculated using Eq. (54) and is listed in Table 7. Since the matrix $\mathbf{K}^{c g}$ is symmetrix and matrix $\mathbf{M}$ of Eq. (17) is diagonal, the analytical (exact) frequencies (in $\mathrm{Hz}$ ) of $\mathbf{M} \ddot{\mathbf{q}}+\mathbf{K}^{c g} \mathbf{q}=\overline{\mathbf{0}}_{51}$ are

$$
\begin{aligned}
& \bar{f}_{1}=\sqrt{\frac{K_{33}^{c g}}{4 \pi^{2} m^{d}}} \quad(\text { axial mode }) ;\left(\bar{f}_{2}=\bar{f}_{3}(\text { bounce mode }), \bar{f}_{4}=\bar{f}_{5}(\text { rock/pitch mode })\right) \\
&=\sqrt{\frac{\left(m^{d} K_{55}^{c g}+I_{d}^{d} K_{11}^{c g}\right)}{8 \pi^{2} m^{d} I_{d}^{d}} \mp \frac{\sqrt{\left(m^{d} K_{55}^{c g}+I_{d}^{d} K_{11}^{c g}\right)^{2}-4 m^{d} I_{d}^{d}\left(K_{11}^{c g} K_{55}^{c g}-K_{15}^{c g} K_{15}^{c g}\right)}}{8 \pi^{2} m^{d} I_{d}^{d}}}
\end{aligned}
$$

where the upper sign (-) is for the bounce mode frequency and the lower sign $(+)$ is for the rock mode frequency. The stiffness matrix elements $K_{33}^{c g}, K_{11}^{c g}, K_{55}^{c g}$ and $K_{15}^{c g}$ are from Eq. (54).

For comparison, the eigenvalues are obtained using QR method of DAMRO-1 program [43] and MATLAB [53], see Table 8. Aini et al. [51,16] conducted impact (hammer) testing of the static spindle assembly normal to its longitudinal axis at the application point of external applied force. They found the fundamental bounce mode in the vertical transverse plane to be in the $0.7-1.2 \mathrm{kHz}$ region, the frequencies band associated with the axial vibrations to be in $0.0-0.7 \mathrm{kHz}$ region and frequency band of rock/pitch mode of assembly to be between $1.2 \mathrm{and} 2.0 \mathrm{kHz}$, see Table 8. Aini et al. [16] assumed constant (linear) bearing stiffness coefficients at the front and rear bearings and used a two-DOF analytical model (discussed in Section 1.3) and calculated bounce and rock/pitch frequencies only with no account for the axial vibration mode, see Table 8 . On the other hand, our proposed model predicts all natural frequencies and is nonlinear (i.e. it does not require preknowledge of the stiffness coefficients) and takes into account gyroscopic effect of the rotating rotor, see Table 9. In [16,51], the experimental natural frequencies of the rotating spindle assembly under no cutting load are obtained from the spectrum of the spindle response. At rotor speed $\Omega_{s}=$ $1000 \mathrm{rpm}$ and bearing axial preload of $100 \mathrm{~N}$, they [16,51] found the bounce and rock/pitch frequencies to be $1050 \mathrm{~Hz}$ and $1590 \mathrm{~Hz}$, respectively. Here $\mathbf{M} \ddot{\mathbf{q}}+\mathbf{G} \dot{\mathbf{q}}+\mathbf{K}^{c g} \mathbf{q}=\overline{\mathbf{0}}_{51}$ is transformed into a first-order system and its matrix $\mathbf{H}=$ 
Table 6

Experimental grinding spindle-bearings assembly parameters, [16,51]

Bearings parameters:

Inner race bore $=0.04 \mathrm{~m}$; Inner race diameter $=0.046 \mathrm{~m}$; Number of balls $=16$;

Outer race diameter $=0.062 \mathrm{~m}$; Inner race radius of curvature $=0.00408 \mathrm{~m}$;

Outer race radius of curvature $=0.00461 \mathrm{~m}$; Ball diameter $=0.00794 \mathrm{~m}$;

Outer race outside diameter $=0.068 \mathrm{~m}$; Unloaded contact angle $=15^{\circ}$;

Bearing width $=0.015 \mathrm{~m}$; Pitch diameter of ball set $=0.054 \mathrm{~m}$;

Bearing axial preload $=100 \mathrm{~N}$; Both bearings are identical.

Spindle parameters:

Shaft diameter between bearings $=0.04 \mathrm{~m}$; Length of shaft $=0.421 \mathrm{~m}$;

Shaft mass, $m=5.5 \mathrm{~kg}$;

Moment of inertia of shaft about $X_{s}$ or $Y_{s}$ axes, $I_{d}=0.05177 \mathrm{~kg} . \mathrm{m}^{2}$

Moment of inertia of shaft about $Z_{s}$ axis, $I_{p}=0.0044 \mathrm{~kg} . \mathrm{m}^{2}$

Position of left bearing from mass center $=0.0875 \mathrm{~m}$;

Position of right bearing from mass center $=0.174 \mathrm{~m}$.

Table 7

Stiffness matrix coefficients of the grinding spindle-bearings assembly for bearing axial preload $=100 \mathrm{~N}$

\begin{tabular}{llll}
\hline $\mathrm{K}_{11}^{c g}=254789125.278$, & $\mathrm{N} / \mathrm{m} ;$ & $\mathrm{K}_{25}^{c g}=-0.0$, & $\mathrm{N} / \mathrm{rad} ;$ \\
$\mathrm{K}_{12}^{c g}=0.0$, & $\mathrm{N} / \mathrm{m} ;$ & $\mathrm{K}_{33}^{c g}=40469147.099$, & $\mathrm{N} / \mathrm{m} ;$ \\
$\mathrm{K}_{13}^{c g}=0.0$, & $\mathrm{N} / \mathrm{m} ;$ & $\mathrm{K}_{34}^{c g}=0.0$, & $\mathrm{N} / \mathrm{rad} ;$ \\
$\mathrm{K}_{14}^{c g}=0.0$, & $\mathrm{N} / \mathrm{rad} ;$ & $\mathrm{K}_{35}^{c g}=-0.0$, & $\mathrm{N} / \mathrm{rad} ;$ \\
$\mathrm{K}_{15}^{c g}=9106715.531$, & $\mathrm{N} / \mathrm{rad} ;$ & $\mathrm{K}_{44}^{c g}=4681646.330$, & $\mathrm{N} . \mathrm{m} / \mathrm{rad} ;$ \\
$\mathrm{K}_{22}^{c g}=254789125.278$, & $\mathrm{N} / \mathrm{m} ;$ & $\mathrm{K}_{45}^{c g}=0.0$, & $\mathrm{N} . \mathrm{m} / \mathrm{rad} ;$ \\
$\mathrm{K}_{23}^{c g}=-0.0$, & $\mathrm{N} / \mathrm{m} ;$ & $\mathrm{K}_{55}^{c g}=4681646.330$, & $\mathrm{N} . \mathrm{m} / \mathrm{rad} ;$ \\
$\mathrm{K}_{24}^{c g}=-9106715.531$, & $\mathrm{N} / \mathrm{rad} ;$ & $\mathrm{K}_{j i}^{c g}=K_{i j}^{c g}$. & \\
\hline
\end{tabular}

Table 8

Natural frequencies $\bar{f}_{k}(\mathrm{~Hz})$ of spindle-ball bearings assembly for $\Omega_{s}=0$, Bearing axial preload $=100 \mathrm{~N}$

\begin{tabular}{ccccc}
\hline a:Exact* & $\begin{array}{c}\text { This } \\
\text { b:Damro-1* }\end{array}$ & $\begin{array}{c}\text { study: a,b,c } \\
\text { c:Matlab }\end{array}$ & $\begin{array}{c}\text { Analytical: } \\
\text { Ref. [16] }\end{array}$ & $\begin{array}{c}\text { Experimental: } \\
\text { Refs [16,51] }\end{array}$ \\
\hline $\bar{f}_{1}: 431.719$ & 431.719 & 431.771 & - & $0.0-700$ band \\
$\bar{f}_{2}: 1012.767$ & 1012.767 & 1012.241 & 910 & $700-1200$ band \\
$\bar{f}_{3}: 1012.767$ & 1012.767 & 1012.241 & & (bounce mode) \\
$\bar{f}_{4}: 1561.536$ & 1561.536 & 1561.421 & 1470 & $1200-2000$ band \\
$\bar{f}_{5}: 1561.536$ & 1561.536 & 1561.421 & & (rock/pitch mode) \\
\hline
\end{tabular}

$\bar{f}_{1}=f^{a}$ (axial natural frequency), * The results are obtained using $2 \pi=8(\arctan 1)$.

Table 9

Natural frequencies $\bar{f}_{k}(\mathrm{~Hz})$ of spindle-ball bearings assembly for $\Omega_{s}=1000 \mathrm{rpm}$, bearing axial preload $=100 \mathrm{~N}$

\begin{tabular}{cccccc}
\hline & $\begin{array}{c}\text { This study: } \\
\text { a: Damro-1 }\end{array}$ & \%err $^{a}$ & b:Matlab & \%err ${ }^{b}$ & $\begin{array}{c}\text { Experimental: } \\
\text { Refs [16,51] }\end{array}$ \\
\hline $\bar{f}_{1}=f^{a}:$ & 431.719 & & 431.771 & & - (axial mode) \\
$\bar{f}_{2}=f_{1}^{B}:$ & 1012.693 & 3.553 & 1012.162 & 3.604 & 1050 (bounce mode) \\
$\bar{f}_{3}=f_{1}^{F}:$ & 1012.841 & & 1012.321 & & \\
$\bar{f}_{4}=f_{2}^{B}:$ & 1560.902 & 1.830 & 1560.785 & 1.837 & 1590 (rock/pitch mode) \\
$\bar{f}_{5}=f_{2}^{F}:$ & 1562.171 & & 1562.058 & & \\
\hline $\operatorname{Ferr}^{a}=\frac{\text { Experimental-(Damro-1) }}{\text { Experimental }} \times 100 ; \%$ err $^{b}=\frac{\text { Experimental-Matlab }}{\text { Experimental }} \times 100$.
\end{tabular}




\section{Table 10}

Natural frequencies $\bar{f}_{k}(\mathrm{~Hz})$ of spindle-ball bearings assembly for $\Omega_{s}=0,1000 \mathrm{rpm}$, bearing axial preload $=100 \mathrm{~N}, \mathbf{K}_{\text {inc }}^{c g}=\operatorname{diag}\left[\begin{array}{llll}\mathrm{K}_{11}^{c g} & \mathrm{~K}_{22}^{c g} & \mathrm{~K}_{33}^{c g} & \mathrm{~K}_{44}^{c g}\end{array}\right.$ $\mathrm{K}_{55}^{c g}$ ]

\begin{tabular}{cccc}
\hline $\begin{array}{c}\text { Damro-1 } \\
0 \mathrm{rpm}\end{array}$ & & $\begin{array}{c}\text { Damro-1 } \\
1000 \mathrm{rpm}\end{array}$ & \%error \\
\hline $\bar{f}_{1}: 431.719$ & $\bar{f}_{1}:$ & 431.719 & \\
$\bar{f}_{2}: 1083.251$ & $\bar{f}_{2}=f_{1}^{B}:$ & 1083.251 & -3.167 \\
$\bar{f}_{3}: 1083.251$ & $\bar{f}_{3}=f_{1}^{F}:$ & 1083.251 & \\
$\bar{f}_{4}: 1513.493$ & $\bar{f}_{4}=f_{2}^{B}:$ & 1512.785 & 4.856 \\
$\bar{f}_{5}: 1513.493$ & $\bar{f}_{5}=f_{2}^{F}:$ & 1514.201 & \\
\hline \multicolumn{4}{c}{ \%error $=\frac{\text { Experimental-(Damro-1) }}{\text { Experimental }} \times 100$, ex- } \\
perimental values are listed in Table 9.
\end{tabular}

$\left[\begin{array}{cc}\overline{\mathbf{0}}_{55} & \mathbf{I}_{55} \\ -\mathbf{M}^{-1} \mathbf{K}^{c g} & -\mathbf{M}^{-1} \mathbf{G}\end{array}\right]$ is solved using the QR method of DAMRO-1 and the MATLAB line command eig $(\mathbf{H})$ for eigenvalues. Table 9 compares our analytical results to the experimental results of Refs [16,51]. $\mathrm{f}_{1}^{F}$ and $\mathrm{f}_{1}^{B}$ are first forward and backward modes. $\mathrm{f}_{2}^{F}$ and $\mathrm{f}_{2}^{B}$ are second forward and backward modes. Observe that the natural frequency in axial direction $\left(\bar{f}_{1}\right)$ is independent of the spindle speed $\left(\Omega_{s}\right)$. Also, Aini [51] experimentally observed that the axial frequency band $(0.0-0.7 \mathrm{KHz})$ is independent of $\Omega_{s}$. As can be seen the proposed analytical model produces results with excellent agreement with the experimental ones. Observe that the spindle rotation (gyroscopic effect) has small effect. Also Aini [51] experimentally observed that the spindle gyroscopic effect at $1000 \mathrm{rpm}$ has small effect on the dynamic response.

\subsection{Applications}

\subsubsection{Effect of using the incorrect (diagonal) stiffness matrix, $\mathbf{K}_{\text {inc }}^{c g}$.}

Here, effect of using matrix $\mathbf{K}_{i n c}^{c g}=\operatorname{diag}\left[\begin{array}{lllll}K_{11}^{c g} & K_{22}^{c g} & K_{33}^{c g} & K_{44}^{c g} & K_{55}^{c g}\end{array}\right]$ whose coefficients $K_{i i}^{c g}$ are from Eq. (54) versus the full (correct) stiffness matrix $\mathbf{K}^{c g}$ of Eq. (54) on the spindle response is presented within the limits of a research study. The rotor is loaded by mass center unbalance of eccentricities $\mu_{a}=\mu_{b}=18 \mu \mathrm{m}$ and gravity load of its own weight $(54 \mathrm{~N})$. The damping coefficient $C_{v}^{b}=40 \mathrm{~N} . \mathrm{s} / \mathrm{m}$. The spindle-bearings data are given in Table 6 . If $\mathbf{K}^{c g}$ is used, full damping matrix $\mathbf{D}^{c g}$ is used. If $\mathbf{K}_{i n c}^{c g}$ is used, diagonal damping matrix $\mathbf{D}_{i n c}^{c g}$ is used. Since the inertia matrix $\mathbf{M}$ is diagonal, using diagonal stiffness matrix uncouples the $x$ and $y$ motions from each other and from each of the rotational motions. It makes rotational motions coupled to each other by only the gyroscopic effect of the rotating shaft. As a result, the bounce mode frequency is not affected by inner ring rotation and the rock/pitch mode frequency is affected as can be seen in Table 10 which tabulates natural frequencies when matrix $\mathbf{K}_{i n c}^{c g}$ is used. Comparing Table 10 to Table 9 for $\Omega_{s}=1000 \mathrm{rpm}$ and Table 10 to Table 8 for $\Omega_{s}=0$ rpm where $\Omega_{s}=0.0 \mathrm{rpm} / 1000 \mathrm{rpm}$ refers to execluding/including the rotor gyroscopic effect, we see that using matrix $\mathbf{K}_{i n c}^{c g}$ caused an increase in value of the bounce mode frequency and decrease in value of the rock/pitch mode frequency. The axial mode frequency value is not affected. These trends of change in eigenvalues are observed for different axail preload values, see Figs 8(a) to 8(c) that depict variation in axial, bounce and rock frequencies, respectively. The most affected frequency is the bounce. The natural frequency change is nonlinear and its rate is rapid at lower values of axial preload. As preload increases, the bearings become stiffer and the change rate decreases.

In the next section, effect of using $\mathbf{K}_{i n c}^{c g}$ matrix will be further briefly highlighted (see Fig. 11).

\subsubsection{Time-varying stiffness matrix $\left(\mathbf{K}^{c g}\right)$ versus standard formulation stiffness matrix $\left(\mathbf{K}_{\text {std }}^{c g}\right)$}

If the bearing cage rotation is not considered in the above presented analyses, the stiffness matrix becomes that of the standard formulation and is denoted hereafter by $\mathbf{K}_{s t d}^{c g}$.

For brevity, we present two examples to highlight the contributions of the presented analyses.

In the first example, the spindle-bearings system is loaded by mass unbalance of eccentricties $\mu_{a}=\mu_{b}=18 \mu \mathrm{m}$, gravity load of its own weight $(54 \mathrm{~N})$ and a force of magnitude $203 \mathrm{~N}$ at $9.87^{\circ}$ angle to the positive $X_{s}$ axis in the 


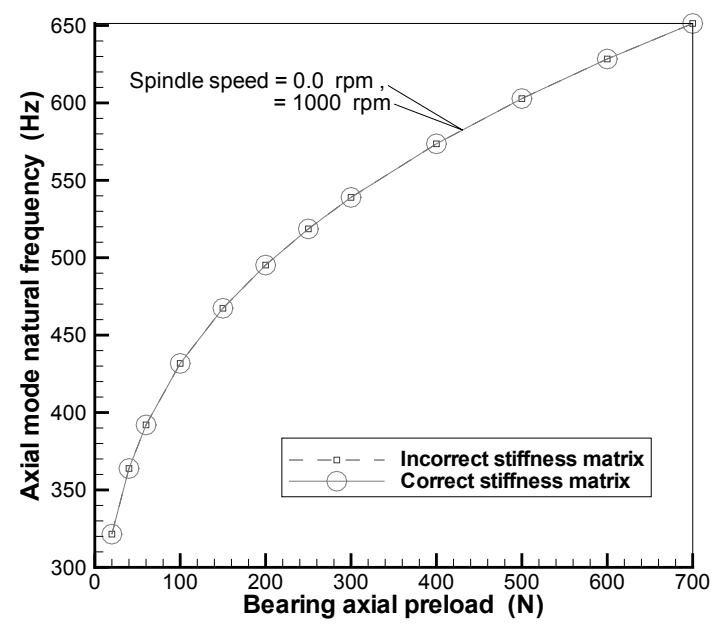

(a)

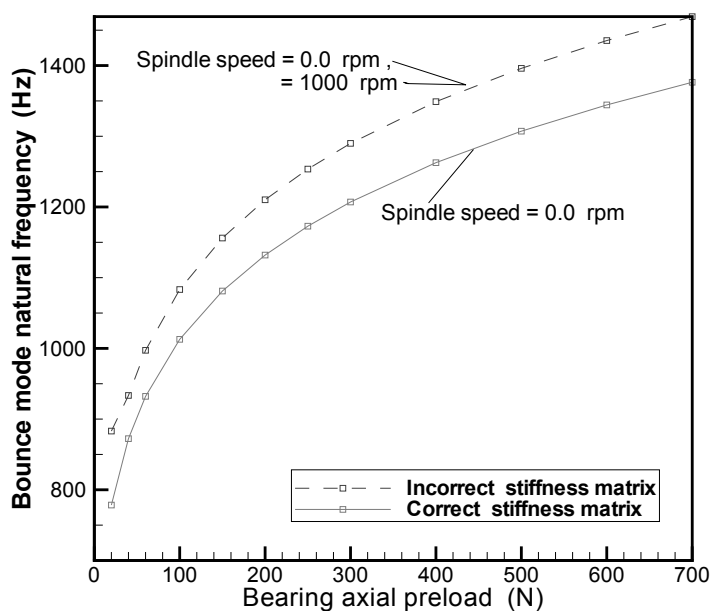

(b)

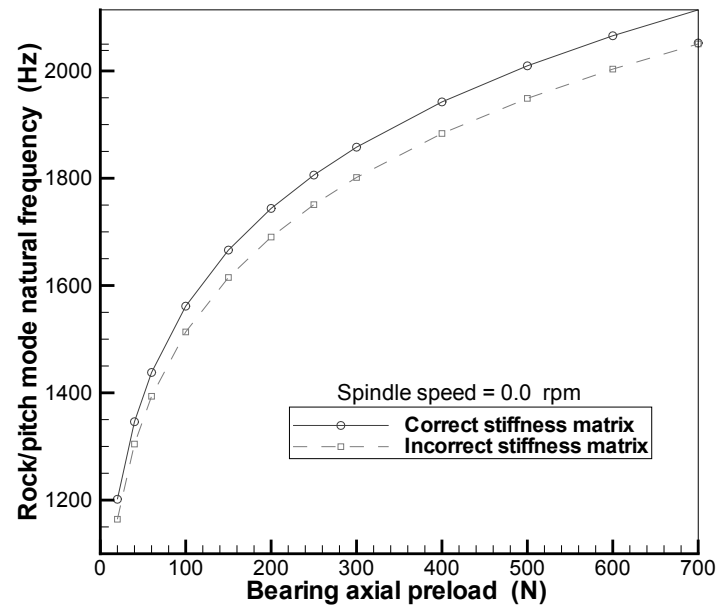

(C)

Fig. 8. Natural frequencies of the machine spindle-bearings assembly vs bearing axial preload with consideration of shaft gyroscopic effect ( $\Omega_{s}=1000 \mathrm{rpm}$ ) and without gyroscopic effect $\left(\Omega_{s}=0.0 \mathrm{rpm}\right.$ ): (a) axail frequency; (b) bounce frequency; (c) rock/pitch frequency. 


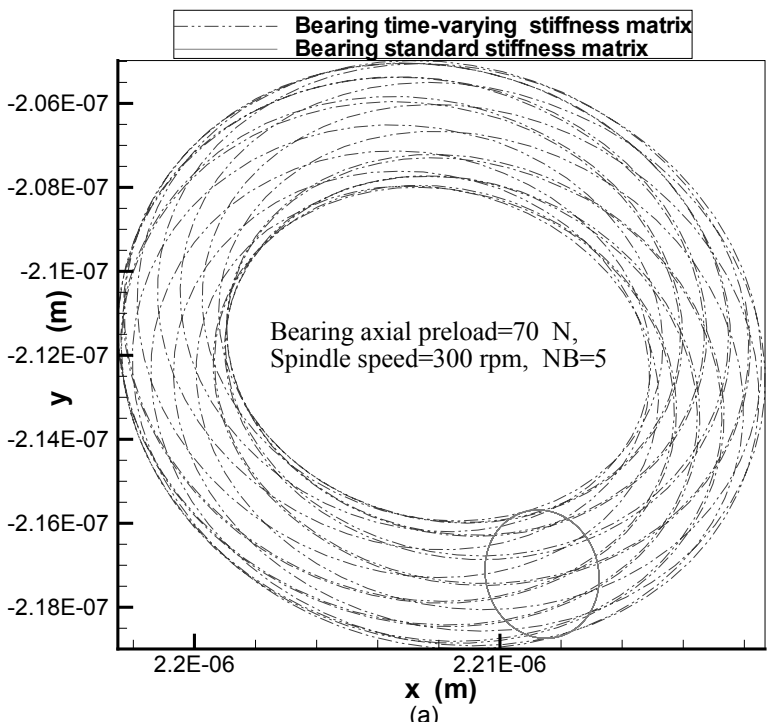

(a)

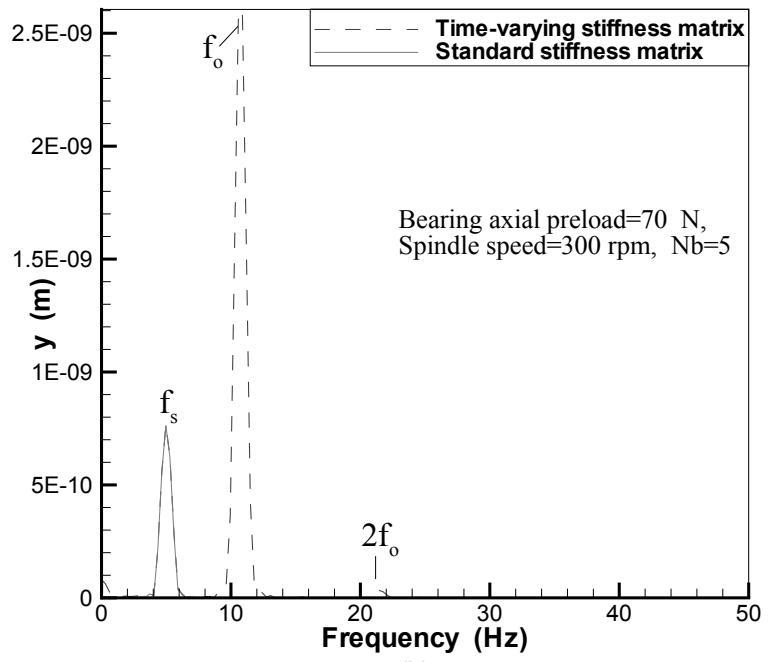

(b)

Fig. 9. (a): Gravity center orbit for time-varying stiffness matrix (dash-dot-dot line) and standard stiffness matrix (solid line). external force $=$ $203 \mathrm{~N}$ force acting in vertical plane at gravity center and is inclined at $9.87 \mathrm{deg}$ to the positive $X_{s}$. $\Omega_{s}=300 \mathrm{rpm}$. mass unbalance eccentricity $\mu_{a}=\mu_{b}=18 \mu \mathrm{m}$. bearing number of balls $\mathrm{Nb}=5$, bearing axial preload $=70 \mathrm{~N}$. (b): FFT of $y$ (vertical) vibration of orbit in (a) for time-varying stiffness matrix (dashed line) and standard stiffness matrix (solid line).

horizontal plane acting at the mass center. (this force could be due to a belt tension force, for example). The bearing axial preload $=70 \mathrm{~N}$, number of balls $\mathrm{Nb}=5$ and damping $c_{v}^{b}=40 \mathrm{~N} . \mathrm{s} / \mathrm{m} . \Omega_{s}=300 \mathrm{rpm}$. Figure 9 (a) shows orbit of the mass center for our time-varying stiffness matrix analyses (dash-dot-dot line) and the standard formulation stiffness (solid line). FFT of the vertical displacement of each case is given in Fig. 9(b). Here the orbit of the $\mathbf{K}^{c g}$ formulation has higher amplitudes and a net like structure (an indication of a multifrequency response). On the other hand, the solid line orbit is a closed curve of lower amplitudes. Spectrum of the $\mathbf{K}^{c g}$ case (dashed line) has the $f_{s}$ (the rotating unbalance frequency), $f_{o}$ and $2 f_{o}$ peaks and FFT of $\mathbf{K}_{s t d}^{c g}$ formulation (solid line) has only one peak at $f_{s}$. Figures 10 (a) and 10(b) depict time domains of the stiffness matrix elements $K_{11}^{c g}$ and $K_{33}^{c g}$, respectively, each for the time-varying matrix (dash-dot-dot line) and the standard formulation matrix (solid line). Figure 10(c) shows time domain of $K_{44}^{c g}$ and its FFT for time-varying stiffness. Here we see that the peak-to-valley variation in the stiffness diagonal coefficients value is small. The FFT of the standard stiffness matrix elements (not shown for brevity) is marked by only a single peak at $f_{s}$. On the otherhand, FFT of the $\mathbf{K}^{c g}$ case is marked by peaks at $f_{s}, \mathrm{n} f_{o}$ ( $\mathrm{n}=1,2,3,4 ; f_{o}=$ outer ring ball passing frequency), see Fig. 10(c). The time domains of the off-diagonal elements $K_{12}^{c g}$ and $K_{35}^{c g}$ are given in Figs $10(\mathrm{~d})$ and 10(e), respectively. Here, the peak-to-valley variation in the stiffness value for time-varying formulation is signifcant. These observations applies to the other off-diagonal elements (not shown). The peak-to-valley variation in the standard stiffness elements is still very small. FFT of the signal $K_{35}^{c g}$ of Fig 10(e) is shown in Fig. 10(f) where we see three peaks at $f_{s}, f_{o}$ and $2 f_{o}$ for $\mathbf{K}^{c g}$ matrix (dash-dot-dot) and only one peak at $f_{s}$ for $\mathbf{K}_{\text {std }}^{c g}$ formulation (solid line). Observe that variation in the stiffness coefficients of the standard $\left(\mathbf{K}_{s t d}^{c g}\right)$ formulation is always periodic of period equals to the rotating unbalance period $\left(1 / f_{s} \mathrm{~s}\right)$.

Next the bearing number of ball is increased to $\mathrm{Nb}=16$ and the rest of operating parameters are the same as above. Figure $10(\mathrm{~g})$ depicts time domain of $K_{35}^{c g}$ for time-varying matrix case only. FFT is shown in Fig. 10(h) for time-varying case (dash line) and standard case (solid line). The time-varying case is marked by two peaks at $f_{s}$ and $f_{o}$. The standard case has only one tone at $f_{s}$. Comparing Fig. 10(g) to Fig. 10(e) we see that increasing the number of balls makes bearing stiffer and as a result the peak-to-valley variation in the stiffness decreases but it is still significant. This observation applies to the other stiffness coefficients, see for example Fig. 10(i) which depicts time domain of $K_{45}^{c g}$ and its spectra where FFT is marked by the tones at $f_{s}, f_{o}$ and $f_{o} \pm f_{s}$.

Figure 11 shows time domain of $K_{35}^{c g}$ for the diagonal (incorrect) time-varying stiffness matrix case (discussed above in Section 3.4.1). This plot is generated using the same data used to obtain Fig. 10(e) for the time-varying stiffness case except that the stiffness matrix is diagonal. Comparing this plot to its counterpart of the correct 


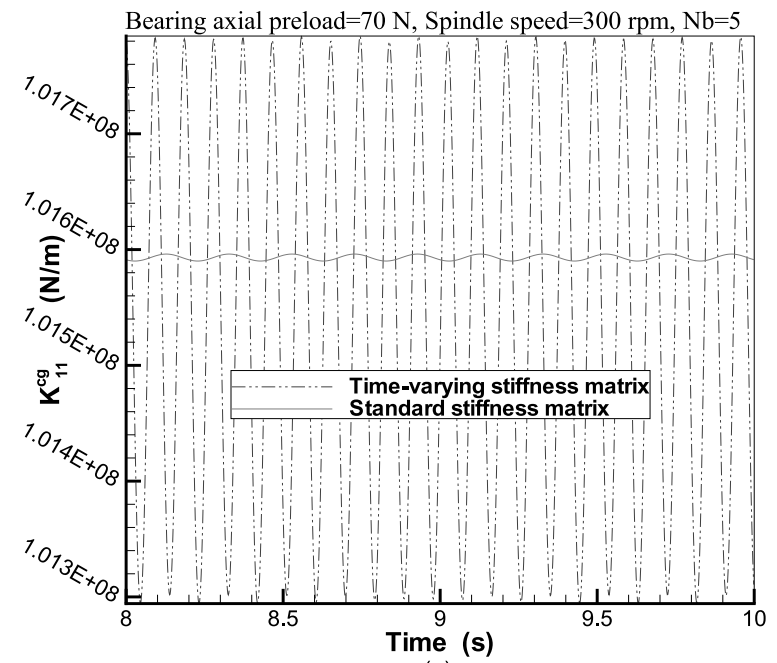

(a)

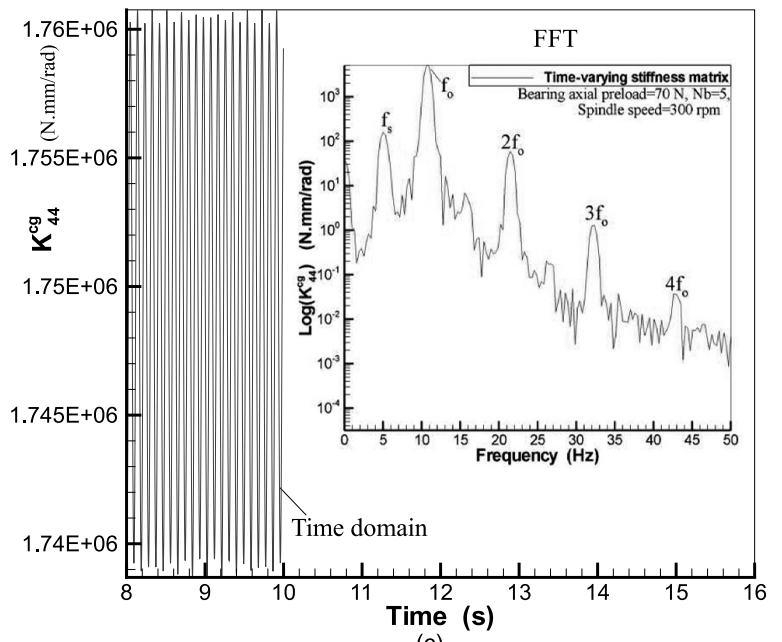

(c)

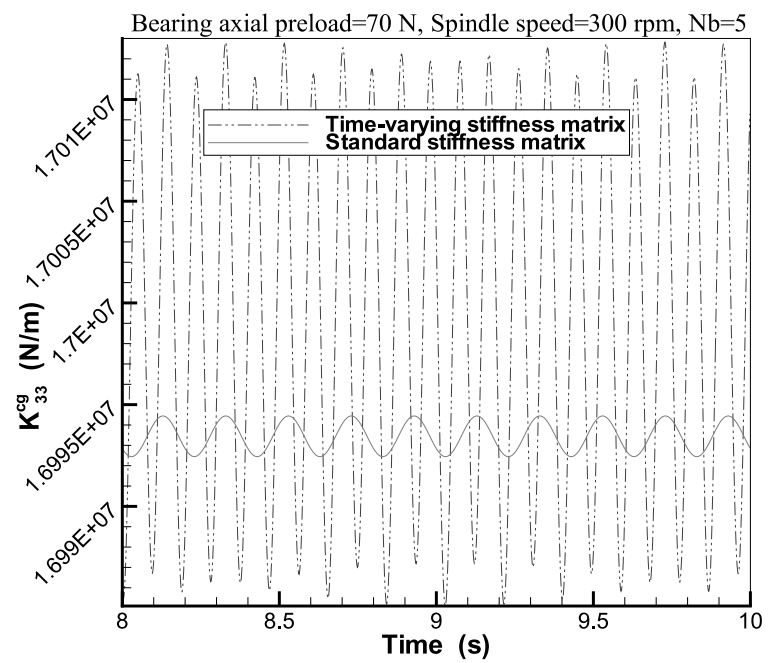

(b)

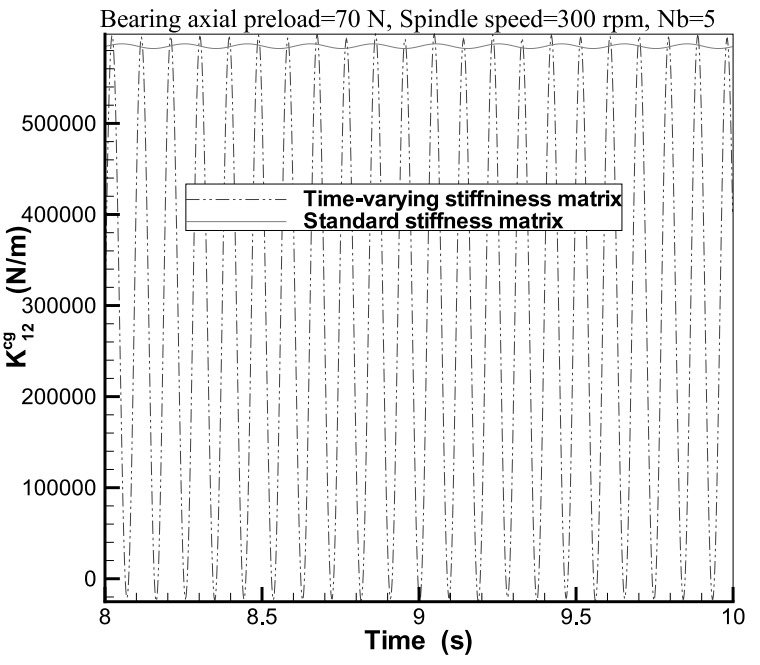

(d)

Fig. 10. (a): Time domain of $K_{11}^{c g}$ for time-varying stiffness matrix (dash-dot-dot line) and standard stiffness matrix (solid line). External load is $203 \mathrm{~N}$ force acting in the vertical plane at gravity center and is inclined at $9.87 \mathrm{deg}$ to the positive $X_{s}$. $\Omega_{s}=300 \mathrm{rpm}$. mass unbalance eccentricity $=18 \mu \mathrm{m} . \mathrm{Nb}=5$, bearing axial preload $=70 \mathrm{~N}$. (b): As in (a) but for $K_{33}^{\mathrm{cg}}$ for time-varying stiffness matrix (dash-dot-dot line) and standard stiffness matrix (solid line). (c): Time domain and FFT of $K_{44}^{c g}$ for time-varying stiffness matrix. Same input data as in (a) (d): As in (a) but for $K_{12}^{c g}$ for time-varying stiffness matrix (dash-dot-dot line) and standard stiffness matrix (solid line). (e): As in (a) but for $K_{35}^{c g}$ for time-varying stiffness matrix (dash-dot-dot line) and standard stiffness matrix (solid line). (f): FFT of $K_{35}^{c g}$ in (e) for time-varying stiffness matrix (dash-dot-dot line) and standard stiffness matrix (solid line). (g): Time domain of $K_{35}^{c g}$ for time-varying stiffness matrix. Same input data as in (a) except that $\mathrm{Nb}=16$. (h): FFT of $K_{35}^{c g}$ in (g) (dashed line) and FFT of its counter part $\left(K_{35}^{c g}\right)$ of standard stiffness matrix (solid line). (i): Time domain and FFT of $K_{45}^{c g}$ for time-varying stiffness matrix. Same input data as in (a) except that $\mathrm{Nb}=16$.

time-varying stiffness (dash-dot-dot line) in Fig. 10(e), we see that using the time-varying diagonal stiffness matrix analysis results in a waveform of lower energy contents compared to the time-varying full matrix waveform. The FFT of plot in Fig. 11 (not shown) is similar to that of Fig. 10(f) for the time-varying stiffness case and amplitude value of its dominant peak $\left(f_{o}\right)$ is 0.132 folds of amplitude value of $f_{o}$ of Fig. 10(f). i.e. response of the diagonal (incorrect) stiffness formulation is of lower energy contents. This observation applies to the rest of the stiffness matrix elements (not shown for brevity). Therefore it is recommended not to use the diagonal $\left(\mathbf{K}_{\text {inc }}^{c g}\right)$ matrix in the vibration analysis of such rotor-bearings systems.

In the second example, the spindle-bearing system is excited by mass unbalnce as above plus a force $F_{c u t}=50 \mathrm{~N}$ in the hrizontal $\left(X_{s}\right)$ direction at the spindle cutting nose. The bearing axial preload $=70 \mathrm{~N}$. For $\mathrm{Nb}=5$, Fig. $12(\mathrm{a})$ 


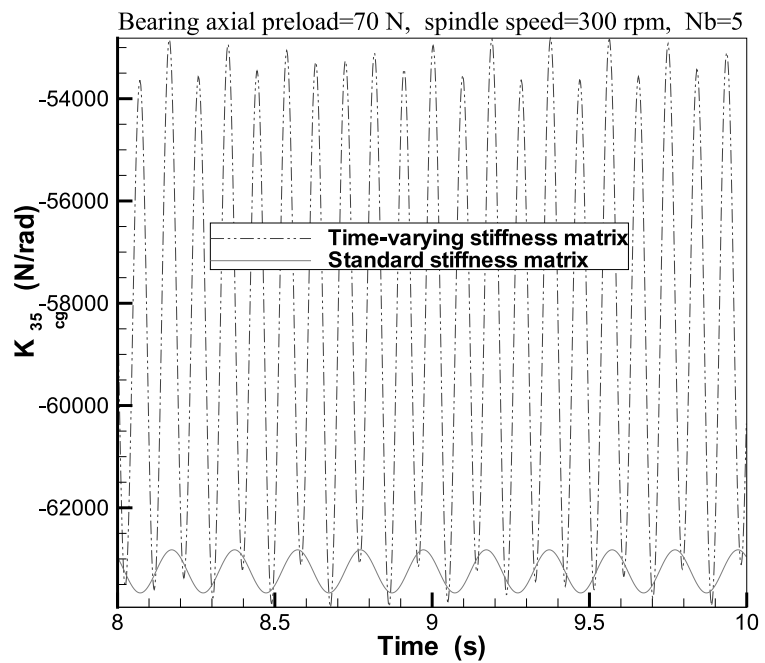

(e)

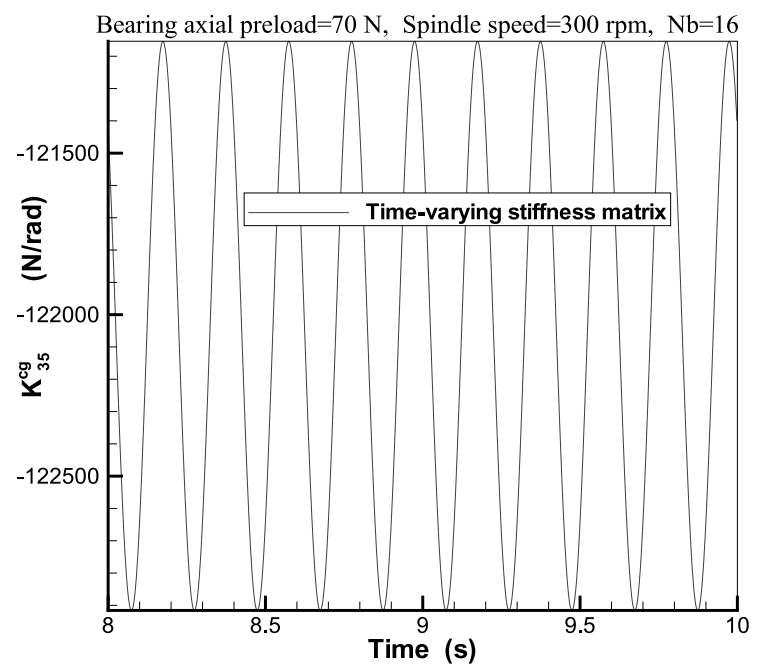

(g)

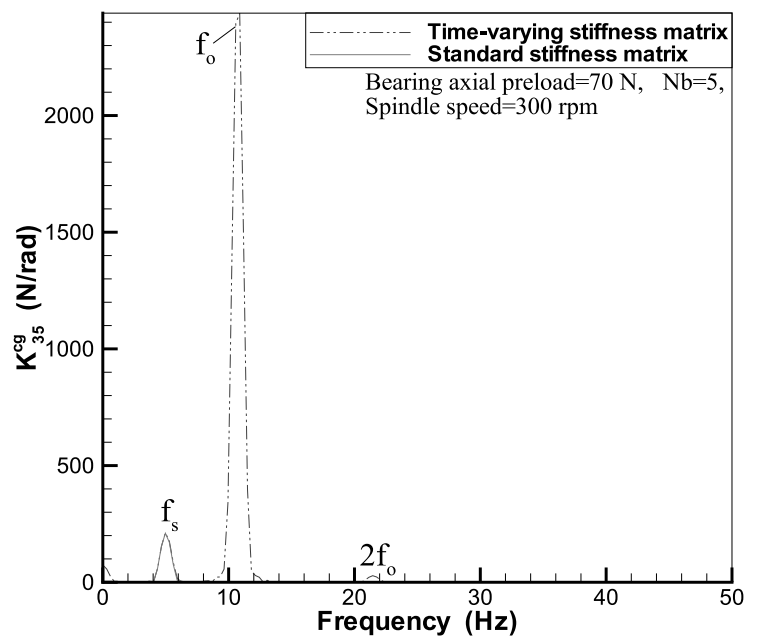

(f)

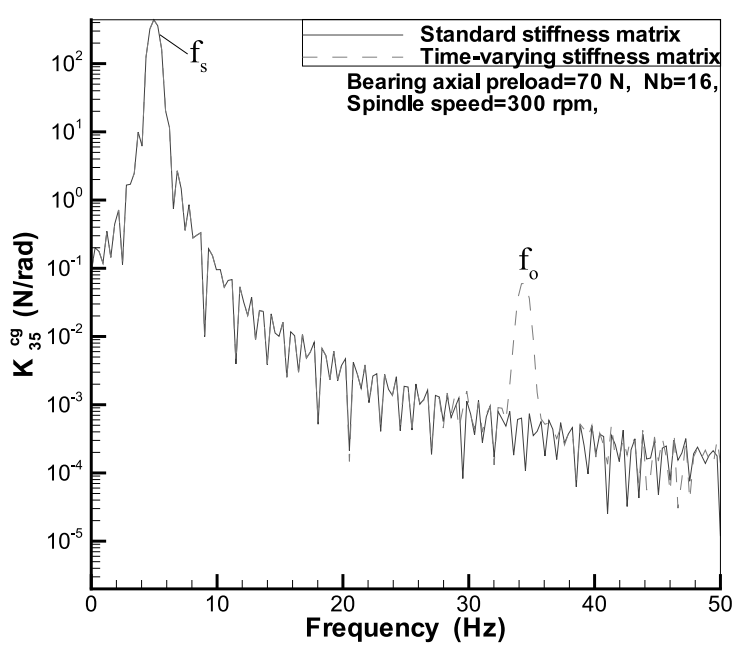

(h)

Fig. 10. continued.

depicts time domain of $K_{35}^{c g}$ and its FFT. The peak-to-valley variation in $K_{35}^{c g}$ is noticable and FFT has peaks at $f_{s}$ (dominant) and $f_{o}$. Time domain of the mass center axial accelration ( $\left.\ddot{z}\right)$ and its FFT are shown in Fig. 12(b). FFT is marked by the $f_{s}, f_{o}, 2 f_{o}$ and $f^{a}$ tones where $f^{a}=279.84 \mathrm{~Hz}$ is owing to the natural frequency of the spindle-bearings assembly in the axial direction. When the number of balls is increased to $\mathrm{Nb}=16$ and the rest of input data are the same as in Fig. 12, Fig. 13 shows time domain of $K_{35}^{c g}$ and its FFT. As a result of increasing the number of balls, the spindle-bearings system becomes more stiff and stiffness becomes periodic. FFT has one visible peak at $f_{s}$.

\section{Conclusions}

1. A lagrangian formulation is developed for computation of the total dynamic stiffness matrix ( $\mathbf{K}^{c g}$ ) and total dynamic damping matrix $\left(\mathbf{D}^{c g}\right)$ of a rigid shaft carrying noncentral rigid disk and supported on angular contact ball bearings. The coefficients of $\mathbf{K}^{c g}$ and $\mathbf{D}^{c g}$ are presented analytically. The formulation is new and takes into account the bearing nonlinearity, variable compliance (i.e. bearing cage rotation) and the bearing axial 


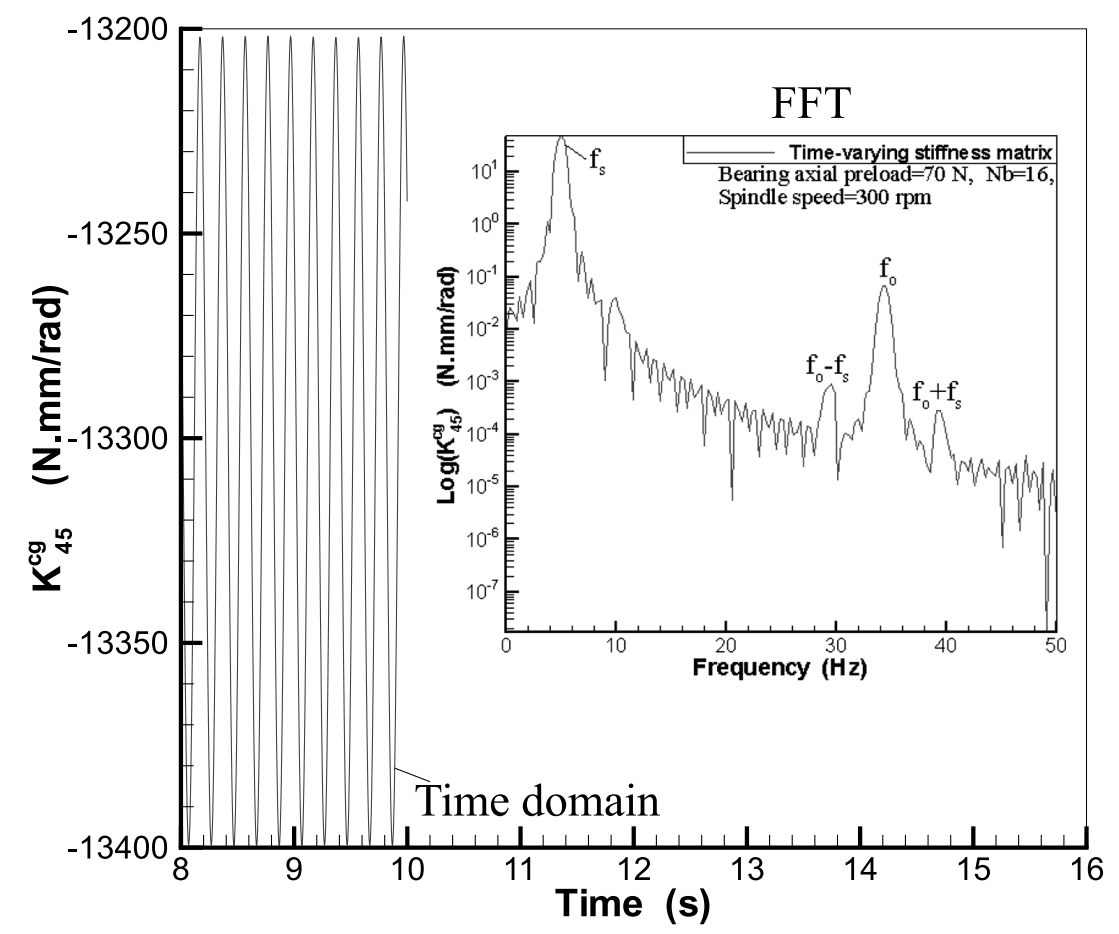

(i)

Fig. 10. continued.

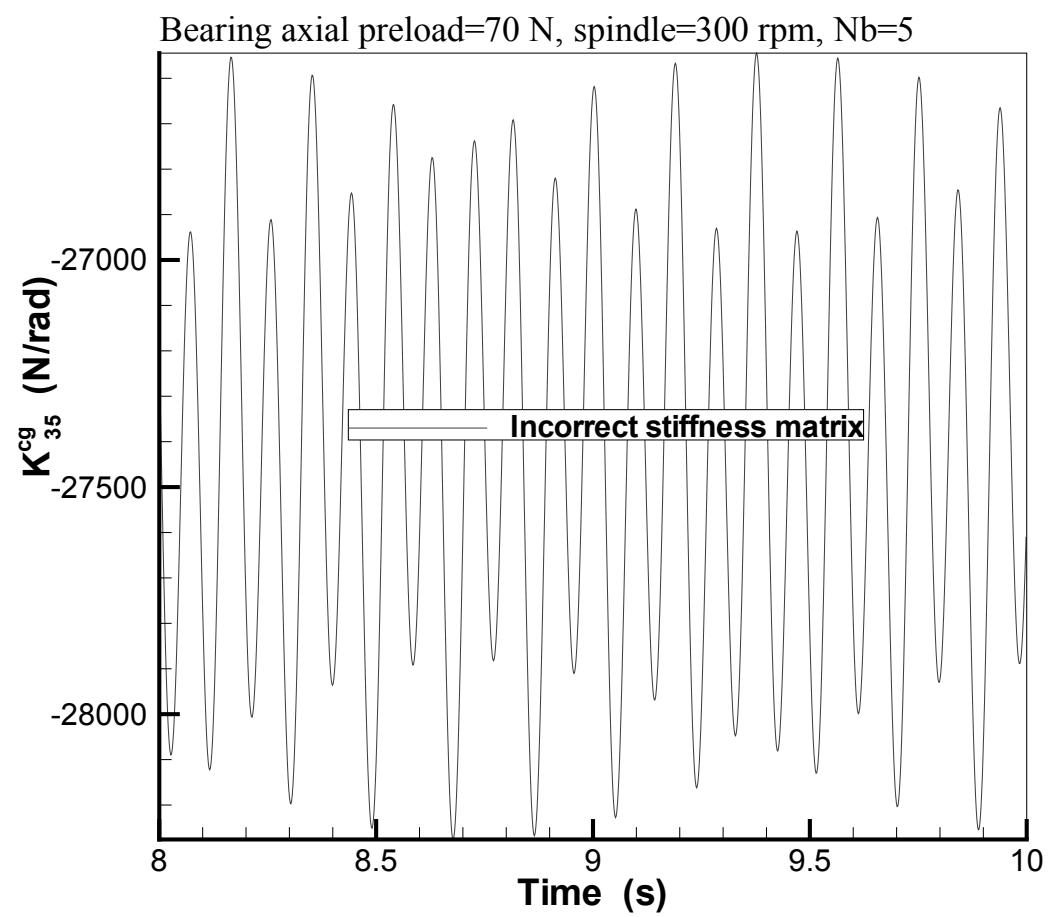

Fig. 11. Time domain of $K_{35}^{c g}$ for the diagnoal (incorrect) total time-varying stiffness matrix (K $\mathbf{K}_{\text {inc }}^{c g}$ ). Same input data as in Fig. 10(e) for the time-varying stiffness case except that the stiffness matrix is diagonal. 


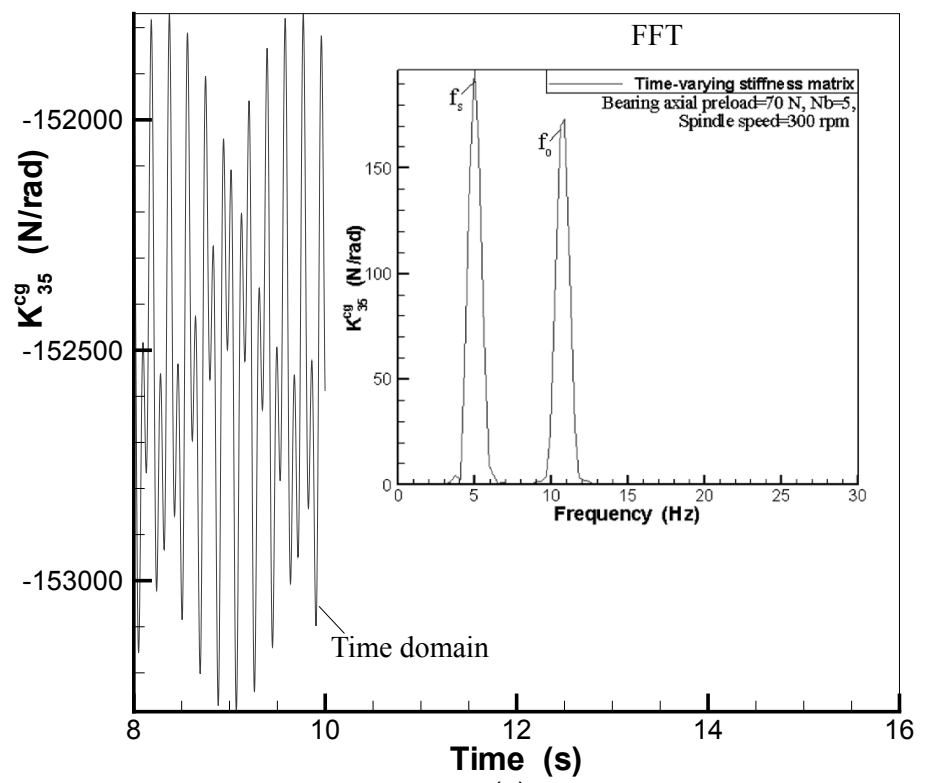

(a)

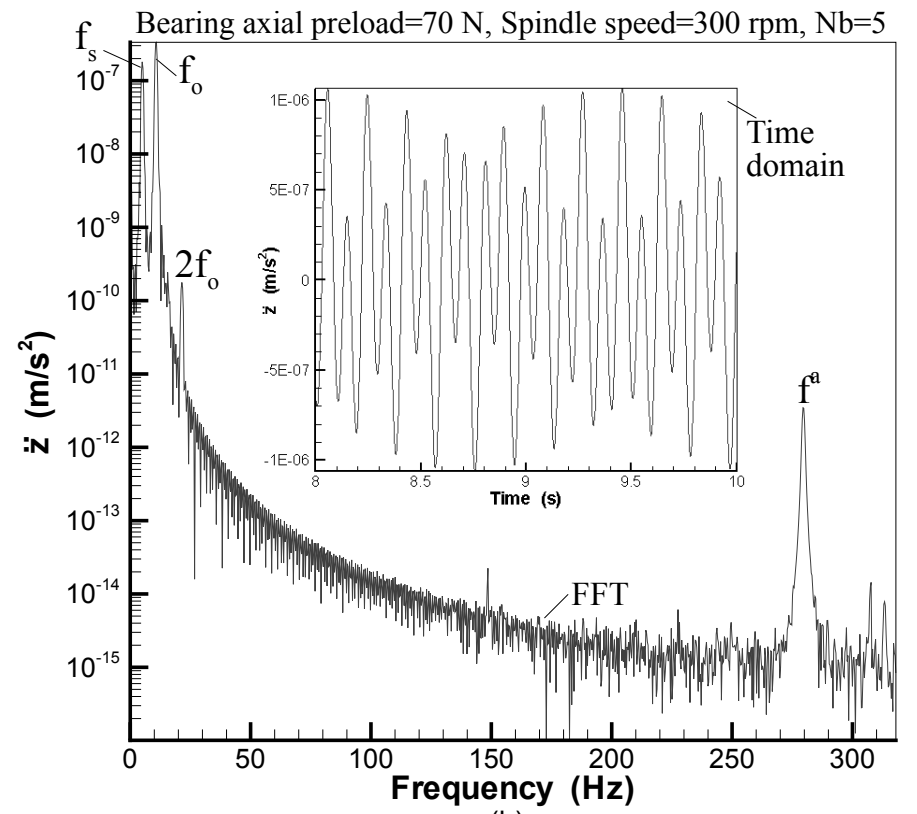

(b)

Fig. 12. Time domain and FFT of $K_{35}^{c g}$ [Fig. 12(a)]; Time domain and FFT of the mass center axial vibration [Fig. 12(b)]. Time varying stiffness matrix is used. $\Omega_{s}=300 \mathrm{rpm}$. Mass eccentricity $\mu_{a}=\mu_{b}=18 \mu \mathrm{m}$. Spindle is subject to a force $F_{c u t}=50 \mathrm{~N}$ in the horizontal direction at its cutting nose. $\mathrm{Nb}=5$. Bearing axial preload $=70 \mathrm{~N}$.

preload. The formulation is verified against existing analytical/experimental results of two-DOF/five-DOF bearing-rigid rotor systems under static/dynamic loads and excellent agreements are obtained.

2. The presented analyses provide effective design tool for the calculation of all the natural frequencies (axial, bounce and rock/pitch) of stationary/rotating machinery supported on angular contact ball bearings with no need for any preknowledge of the bearing stiffness. This closes the gaps in existing analyses on solution of eigen problem of such rotating machinery.

3. Using the rotor-bearings diagonal (incorrect) stiffness matrix $\left(\mathbf{K}_{\text {inc }}^{c g}\right)$ uncouples the three translational motions 


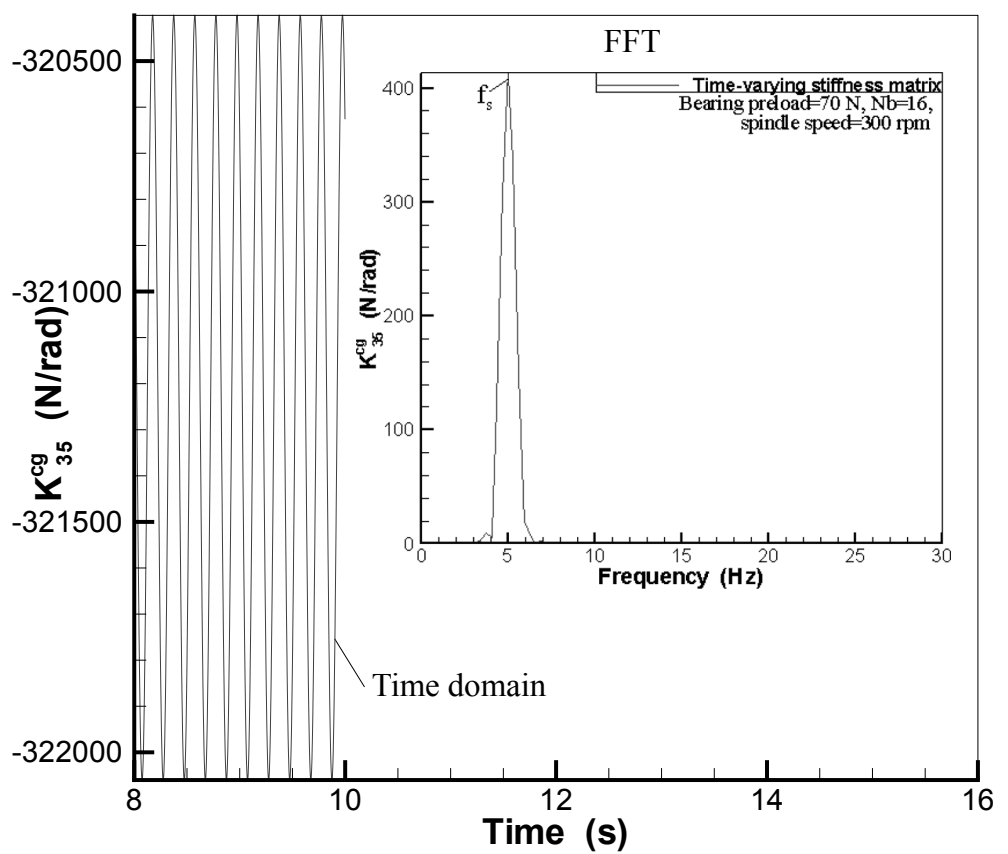

Fig. 13. Time domain and FFT of $K_{35}^{c g}$. Time varying stiffness matrix is used. Same input data as in Fig. 12 except that $\mathrm{Nb}=16$.

from the two rotational motions which in turn become coupled to each other by only the rotating shaft gyroscopic effect. This makes the bounce frequency independent of the rotor speed (gyroscopic) effect and its value increases and vice versa if the full time-varying (correct) stiffness matrix $\left(\mathbf{K}^{c g}\right)$ is used. On the other hand value of the rock frequency decreases when $\mathbf{K}_{i n c}^{c g}$ is used compared to its value when $\mathbf{K}^{c g}$ is used.

These trends of change in the natural frequencies hold for the bearing axial preload range $10 \mathrm{~N}$ to $700 \mathrm{~N}$ studied and the absolute value of change difference increases as the axial preload increases. The axial frequency value is not affected by the rotor gyroscopic effect or the choice of $\mathbf{K}^{c g}$ or $\mathbf{K}_{\text {inc }}^{c g}$ but its value increases nonlinearly as axial preload increases.

4. When the rotor center of gravity is loaded by mass unbalance and a constant force in each of the vertical and horizontal directions and the bearings are axially preloaded, the dominant vibration of each of the $\mathbf{K}^{c g}$ matrix coefficients becomes of higher energy contents compared to that of the $\mathbf{K}_{i n c}^{c g}$ coefficients. Therefore, it is highly recommended not to use the diagonal (incorrect) stiffness matrix in the design and/or analysis of rigid rotor-ball bearings systems to avoid missing important vibration peaks or underestimate of the vibrations amplitudes.

5. When the rotor center of gravity is lodaed as above and the time-varying stiffness formulation is used, the peak-to-valley variation in the stiffness matrix coefficients becomes sgnificant in the off-diagonal elements compared to the diagonal elements. Increasing the number of bearing ball makes spindle more stiff and decreases the paek-to-valley variation of the stiffness coefficients but this variation is still significant and can not be ignored.

When the standard stiffness matrix $\left(\mathbf{K}_{s t d}^{c g}\right.$ ) formulation is used, the peak-to-valley variation in the stiffness coefficients becomes very small (compared to variation in the $\mathbf{K}^{c g}$ case) and always periodic of period equals to the rotating mass unbalance period.

6. When the rotor is unbalanced, the bearings are axially preloaded and the spindle is under a force in the horizontal direction at its cutting nose, the above conclusions regarding peak-to-valley variations in the stiffness coefficients still hold. Therefore it is recommended to treat the spindle-bearings stiffness matrix to be time-varying. 


\section{Acknowledgment}

The author thanks the paper reviewers for their contributing comments and suggestions.

\section{Appendix A: Angular contact ball bearing stiffness matrix $\mathbf{K}^{\mathbf{b}}$}

$$
\begin{aligned}
& K_{11}^{b}=\frac{\partial F_{e x}^{b}}{\partial x^{b}}=\sum_{j=1}^{N b} P_{j}^{b}\left(\frac{n \cos ^{2} \alpha_{j}}{S_{j}-S_{o}}+\frac{\sin ^{2} \alpha_{j}}{S_{j}}\right) \cos ^{2}\left(\Omega_{c} t+\phi_{j}\right) . \\
& K_{12}^{b}=\frac{\partial F_{e x}^{b}}{\partial y^{b}}=\frac{1}{2} \sum_{j=1}^{N b} P_{j}^{b}\left(\frac{n \cos ^{2} \alpha_{j}}{S_{j}-S_{o}}+\frac{\sin ^{2} \alpha_{j}}{S_{j}}\right) \sin 2\left(\Omega_{c} t+\phi_{j}\right) . \\
& K_{13}^{b}=\frac{\partial F_{e x}^{b}}{\partial z^{b}}=\frac{1}{2} \sum_{j=1}^{N b} P_{j}^{b}\left(\frac{n}{S_{j}-S_{o}}-\frac{1}{S_{j}}\right) \sin 2 \alpha_{j} \cos \left(\Omega_{c} t+\phi_{j}\right) . \\
& K_{14}^{b}=\frac{\partial F_{e x}^{b}}{\partial \theta_{x}^{b}}=\frac{r_{m}}{4} \sum_{j=1}^{N b} P_{j}^{b}\left(\frac{n}{S_{j}-S_{o}}-\frac{1}{S_{j}}\right) \sin 2 \alpha_{j} \sin 2\left(\Omega t_{c}+\phi_{j}\right) . \\
& K_{15}^{b}=\frac{\partial F_{e x}^{b}}{\partial \theta_{y}^{b}}=\frac{r_{m}}{2} \sum_{j=1}^{N b} P_{j}^{b}\left(\frac{-n}{S_{j}-S_{o}}+\frac{1}{S_{j}}\right) \sin 2 \alpha_{j} \cos ^{2}\left(\Omega_{c} t+\phi_{j}\right) . \\
& K_{22}^{b}=\frac{\partial F_{e y}^{b}}{\partial y^{b}}=\sum_{j=1}^{N b} P_{j}^{b}\left(\frac{n \cos ^{2} \alpha_{j}}{S_{j}-S_{o}}+\frac{\sin ^{2} \alpha_{j}}{S_{j}}\right) \sin ^{2}\left(\Omega_{c} t+\phi_{j}\right) . \\
& K_{23}^{b}=\frac{\partial F_{e y}^{b}}{\partial z^{b}}=\frac{1}{2} \sum_{j=1}^{N b} P_{j}^{b}\left(\frac{n}{S_{j}-S_{o}}-\frac{1}{S_{j}}\right) \sin 2 \alpha_{j} \sin \left(\Omega_{c} t+\phi_{j}\right) . \\
& K_{24}^{b}=\frac{\partial F_{e y}^{b}}{\partial \theta_{x}^{b}}=\frac{r_{m}}{2} \sum_{j=1}^{N b} P_{j}^{b}\left(\frac{n}{S_{j}-S_{o}}-\frac{1}{S_{j}}\right) \sin 2 \alpha_{j} \sin ^{2}\left(\Omega_{c} t+\phi_{j}\right) . \\
& K_{25}^{b}=\frac{\partial F_{e y}^{b}}{\partial \theta_{y}^{b}}=\frac{r_{m}}{4} \sum_{j=1}^{N b} P_{j}^{b}\left(\frac{-n}{S_{j}-S_{o}}-\frac{1}{S_{j}}\right) \sin 2 \alpha_{j} \sin 2\left(\Omega_{c} t+\phi_{j}\right) . \\
& K_{33}^{b}=\frac{\partial F_{e z}^{b}}{\partial z^{b}}=\sum_{j=1}^{N b} P_{j}^{b}\left(\frac{n \sin ^{2} \alpha_{j}}{S_{j}-S_{o}}+\frac{\cos ^{2} \alpha_{j}}{S_{j}}\right) . \\
& K_{34}^{b}=\frac{\partial F_{e z}^{b}}{\partial \theta_{x}^{b}}=r_{m} \sum_{j=1}^{N b} P_{j}^{b}\left(\frac{n \sin ^{2} \alpha_{j}}{S_{j}-S_{o}}+\frac{\cos ^{2} \alpha_{j}}{S_{j}}\right) \sin \left(\Omega_{c} t+\phi_{j}\right) . \\
& K_{35}^{b}=\frac{\partial F_{e z}^{b}}{\partial \theta_{y}^{b}}=r_{m} \sum_{j=1}^{N b} P_{j}^{b}\left(\frac{-n \sin ^{2} \alpha_{j}}{S_{j}-S_{o}}-\frac{\cos ^{2} \alpha_{j}}{S_{j}}\right) \cos \left(\Omega_{c} t+\phi_{j}\right) . \\
& K_{44}^{b}=\frac{\partial M_{e x}^{b}}{\partial \theta_{x}^{b}}=r_{m}^{2} \sum_{j=1}^{N b} P_{j}^{b}\left(\frac{n \sin ^{2} \alpha_{j}}{S_{j}-S_{o}}+\frac{\cos ^{2} \alpha_{j}}{S_{j}}\right) \sin ^{2}\left(\Omega_{c} t+\phi_{j}\right)
\end{aligned}
$$




$$
\begin{aligned}
& K_{45}^{b}=\frac{\partial M_{e x}^{b}}{\partial \theta_{y}^{b}}=\frac{r_{m}^{2}}{2} \sum_{j=1}^{N b} P_{j}^{b}\left(\frac{-n \sin ^{2} \alpha_{j}}{S_{j}-S_{o}}-\frac{\cos ^{2} \alpha_{j}}{S_{j}}\right) \sin 2\left(\Omega_{c} t+\phi_{j}\right) . \\
& K_{55}^{b}=\frac{\partial M_{e y}^{b}}{\partial \theta_{y}^{b}}=r_{m}^{2} \sum_{j=1}^{N b} P_{j}^{b}\left(\frac{n \sin ^{2} \alpha_{j}}{S_{j}-S_{o}}+\frac{\cos ^{2} \alpha_{j}}{S_{j}}\right) \cos ^{2}\left(\Omega_{c} t+\phi_{j}\right) .
\end{aligned}
$$

$K_{j i}^{b}=K_{i j}^{b} . n=1.5$ for ball bearings.

\section{Appendix B: Angular contact ball bearing damping matrix $\mathrm{D}^{\mathrm{b}}$}

$$
\begin{aligned}
& D_{11}^{b}=\frac{\partial F_{d x}^{b}}{\partial \dot{x}^{b}}=C_{v}^{b} \sum_{j=1}^{N b} \cos ^{2} \alpha_{j} \cos ^{2}\left(\Omega_{c} t+\phi_{j}\right) . \\
& D_{12}^{b}=\frac{\partial F_{d x}^{b}}{\partial \dot{y}^{b}}=\frac{1}{2} C_{v}^{b} \sum_{j=1}^{N b} \cos ^{2} \alpha_{j} \sin 2\left(\Omega_{c} t+\phi_{j}\right) . \\
& D_{13}^{b}=\frac{\partial F_{d x}^{b}}{\partial \dot{z}^{b}}=\frac{1}{2} C_{v}^{b} \sum_{j=1}^{N b} \sin ^{2} \alpha_{j} \cos \left(\Omega_{c} t+\phi_{j}\right) . \\
& D_{14}^{b}=\frac{\partial F_{d x}^{b}}{\partial \dot{\theta}_{x}^{b}}=\frac{r_{m}}{4} C_{v}^{b} \sum_{j=1}^{N b} \sin 2 \alpha_{j} \sin 2\left(\Omega_{c} t+\phi_{j}\right) . \\
& D_{15}^{b}=\frac{\partial F_{d x}^{b}}{\partial \dot{\theta}_{y}^{b}}=-\frac{r_{m}}{2} C_{v}^{b} \sum_{j=1}^{N b} \sin 2 \alpha_{j} \cos ^{2}\left(\Omega_{c} t+\phi_{j}\right) . \\
& D_{33}^{b}=\frac{\partial F_{d y}^{b}}{\partial \dot{y}^{b}}=C_{v}^{b} \sum_{j=1}^{N b} \cos ^{2} \alpha_{j} \sin { }^{2}\left(\Omega_{c} t+\phi_{j}\right) . \\
& D_{23}^{b}=\frac{\partial F_{d y}^{b}}{\partial \dot{z}^{b}}=\frac{1}{2} C_{v}^{b} \sum_{j=1}^{N b} \sin 2 \alpha_{j} \sin \left(\Omega_{c} t+\phi_{j}\right) . \\
& D_{24}^{b}=\frac{\partial F_{d y}^{b}}{\partial \dot{\theta}_{x}^{b}}=\frac{r_{m}^{b}}{2} C_{v}^{b} \sum_{j=1}^{N b} \sin 2 \alpha_{j} \sin { }^{2}\left(\Omega_{c} t+\phi_{j}\right) . \\
& \frac{\partial \dot{\theta}_{y}^{b}}{b}=-\frac{r_{m}}{4} C_{v}^{b} \sum_{j=1}^{N b} \sin 2 \alpha_{j} \sin 2\left(\Omega_{c} t+\phi_{j}\right) . \\
& \left.D_{j=1}^{N b} \sin ^{2} \alpha_{j} \sin \Omega_{c} t+\phi_{j}\right) .
\end{aligned}
$$




$$
\begin{gathered}
D_{35}^{b}=\frac{\partial F_{d z}^{b}}{\partial \dot{\theta}_{y}^{b}}=-r_{m} C_{v}^{b} \sum_{j=1}^{N b} \sin ^{2} \alpha_{j} \cos \left(\Omega_{c} t+\phi_{j}\right) \\
D_{44}^{b}=\frac{\partial M_{d x}^{b}}{\partial \dot{\theta}_{x}^{b}}=r_{m}^{2} C_{v}^{b} \sum_{j=1}^{N b} \sin ^{2} \alpha_{j} \sin ^{2}\left(\Omega_{c} t+\phi_{j}\right) . \\
D_{45}^{b}=\frac{\partial M_{d x}^{b}}{\partial \dot{\theta}_{y}^{b}}=\frac{-r_{m}^{2}}{2} C_{v}^{b} \sum_{j=1}^{N b} \sin ^{2} \alpha_{j} \sin 2\left(\Omega_{c} t+\phi_{j}\right) . \\
D_{55}^{b}=\frac{\partial M_{d y}^{b}}{\partial \dot{\theta}_{y}^{b}}=r_{m}^{2} C_{v}^{b} \sum_{j=1}^{N b} \sin ^{2} \alpha_{j} \cos ^{2}\left(\Omega_{c} t+\phi_{j}\right) . \\
D_{i j}^{b}=D_{j i}^{b} .
\end{gathered}
$$

\section{References}

[1] M.A. Alfares and A.A. Elsharkawy, Effects of axial preloading of angular contact ball bearings on the dynamics of a grinding machine spindle system, Journal of Materials Processing Technology 136 (2003), 48-59.

[2] R. Aini, H. Rahnejat and R. Gohar, A five degrees of freedom analysis of vibrations in precision spindles, Int J Mach Tools Manufact 30(1) (1990), 1-18.

[3] G.P. Adams and Z.H. Qin, Compression load transmission in screw compressors, Journal of Sound and Vibration 207(5) (1997), 671-691.

[4] A.O. Gibson, J.L. Stein and J.F. Tu, A thermomechanical model of machine tool spindles for use in the design of reconfigurable angular contact spindle bearing load control systems, ASME Proceedings Manufacturing Science and Engineering Division MED 1 (1997), 23-30.

[5] A.B. Jones, A general theory for elastically constrained ball and radial roller bearings under arbitrary load and speed conditions, ASME Journal of Basic Engineering June (1960), 309-320.

[6] J.M. De Mul, J.M. Vree and D.A. Mass, Equilibrium and associated load distribution in ball and roller bearings loaded in five degrees of freedom while neglecting friction - Part I: General theory and application to ball bearings, ASME Journal of Tribology 111 (1989), $142-148$.

[7] T.C. Lim and R. Singh, Vibration transmission through rolling element bearings, part I: bearing stiffness formulation, Journal of Sound and Vibration 139(2) (1990), 179-199.

[8] E. Muhlner, Integrated analysis of ball bearings and structures, Ph.D. thesis, Technical University of Vienna (1997).

[9] P. Dietl, Damping and stiffness characteristics of rolling element bearings-theory and experiment, Ph.D. Thesis, Technical University of Vienna (1997).

[10] L. Houpert, Prediction of bearing and housing performance, Proceeding of the Rolling Bearing Practice Today Seminar, IMechE,, London (1995).

[11] L. Houpert, A uniform analytical approach for ball and roller bearings calculations, ASME Journal of Tribology 119 (1997), 851-858.

[12] S. Sjoval, The load distribution within ball and roller bearing under given external radial and axial load, Teknsik Tidskrift, Mekanik Hfte 9 (1933) 97-102.

[13] X. Hernot, M. Sartor and J. Guillot, Calculation of the stiffness matrix of angular contact ball bearings by using analytical approach, ASME Journal of Mechanical Design 122 (2000), 83-90.

[14] D.R. Houser, W. Young, G.L. Kinzel and M. Rajab, Force tramsmissibility through rolling contact bearings, 7th IMAC, Las Vegas, Nevada, 147-153.

[15] G. Sun, A.B. Palazzolo, A. Provenza and G. Montague, Detailed ball bearing model for magnetic suspension auxiliary services. Journal of Sound and Vibration 269 (2004), 933-963.

[16] R. Aini, H. Rahnejat and R. Gohar, An experimental investigation into bearing-induced spindle vibration, Proc. Instn. Mech. Engrs., Part C:J. Mechanical Engineering Science 209 (1995), 107-114.

[17] N. Akturk, Dynamics of a rigid shaft supported by angular contact ball bearings, Ph.D. Thesis, Imerial College of Science, Technology and Medicine, University of London (1993).

[18] N. Akturk, Some characteristic parameters affecting the natural frequency of a rotating shaft supported by defect-free ball bearings, Proc Instn Mech Engrs Part K.J. Multibody Dynamics 217 (2003), 145-151.

[19] N. Lynagh, H. Rahnejat, M. Ebrahimi and R. Aini, Bearing induced vibration in precision high speed routing spindles, International Journal of Machine Tools and Manufacture 40 (2000), 561-577.

[20] S. Vafaei, H. Rahnejat and R. Aini, Vibration monitoring of high speed spindles using spectral analysis techniques, International Journal of Machine Tools and Manufacture 42 (2002), 1223-1234.

[21] B. Mevel and J.L. Guyader, Routes to chaos in ball bearings, Journal of Sound and Vibration 162 (1993), $471-487$.

[22] B. Mevel and J.L. Guyader, Experiments on routes to chaos in ball bearings, Journal of Sound and Vibration 318 (2008), $549-564$. 
[23] H. Tamura and Y. Tsuda, On the spring characteristics of a ball bearing, Bulletin of the JSME 23(180) (1980), 961-969.

[24] H. Shimizu and H. Tamura, Vibration of rotor based on ball bearing. 1st report: static stiffness of ball bearings, Bull Jap Soc Mech Engrs 9(35) (1966), 524-536.

[25] H. Tamura and H. Shimizu, Vibration of rotor based on ball bearing. 2nd report: static stiffness of ball bearings containing a small number of balls, Bull Jap Soc Mech Engrs 10(41) (1967), 763-775.

[26] H. Tamura and H. Shimizu, Vibration of rotor based on ball bearing. 3rd report: static stiffness of ball bearings containing a large number of balls, Bull Jap Soc Mech Engrs 11(47) (1967), 825-837.

[27] T. Yamamoto and Y. Ishida, The particular vibration phenomena due to ball bearings at the majors critical speeds, Bull Jap Soc Mech Engrs 17(103) (1974), 59-67.

[28] H.R. El-Sayed, Stiffness of deep-groove ball bearings, Wear 6 (1980), 89-94.

[29] M.F. White, Rolling element bearing vibration transfer characteristics: effect of stiffness, ASME, Journal of Applied Mechanics 46 (1979), 677-684.

[30] F.M.A. El-Saeidy, Rotating machinery dynamics simulation. I: Rigid systems with ball bearing nonlinearities and outer ring ovality under rotating unbalance excitation, Journal of The Acoustical Society of America 107(2) (2000), 851-860.

[31] H. Walford and B. Stone, The measurement of the radial stiffness of rolling elements bearings under oscillating conditions, Proc. Instn. Mech. Engrs., Part C: J. Mechanical Science Engineering 22(4) (1980), 175-181.

[32] G.H. Jang and S.W. Jeong, Stability analysis of a rotating system due to the effect of a ball bearing waviness, ASME Journal of Tribology 125 (2003), 91-101.

[33] H. Liew and T. Lim, Analysis of time-varying rolling element bearing characteristics, Journal of Sound and Vibration 283 (2005), 1163-1179.

[34] E. Yahland, A linear theory of vibrations caused by ball bearings with form errors operating at moderate speed, ASME Journal of Tribology 114 (1992), 348-359.

[35] . R. Zeillinger and H. Kottritsch, Damping in a rolling arrangement, SKF Business and Technology Magazine (Evolution) 10 (1996), 27-30.

[36] C. Natraj and S.P. Harsha, The effect of bearing cage run-out on the nonlinear dynamics of a rotating shaft, Commuminations in Nonlinear Science and Numerical Simulation 13 (2008), 822-838.

[37] E. Kramer, Dynamics of Rotor and Foundations, New York: Springer-Verlag (1993).

[38] M.O.T. Cole, P.S. Keogh and C.R. Burrows, The dynamic behavior of a rolling element auxiliary bearing following rotor impact, ASME, Journal of Tribology 124 (2002), 406-413.

[39] J.A. Wensing and G.C. Nijen, 2-dimensional computational model for vibration analysis of waviness in rolling bearing applications, C500/136 IMechE (1996), 371-382.

[40] P. Dietl, J. Wensing and G.C. Nijen, Rolling bearing damping for dynamic analysis of multi-body systems-experimental and theoretical results, Proc Inst Engrs, Part K 214, (2000), 33-43.

[41] F.M.A. El-Saeidy, Finite element modeling of a rotor shaft rolling bearings system with consideration of bearing nonlinearities, Journal of Vibration and Control 4 (1998), 541-602.

[42] T.A. Harris, Rolling bearing Analysis, New York John Wiley and Sons (1991).

[43] F.M.A. El-Saeidy, DAMRO-1: A General Purpose Finite Element Program, (1993).

[44] M. Keskiniva, Simulation and balancing of flexible rotors in terms of semidefinite modal coordinates, Doctoral thesis, Tampere University (1997).

[45] B.J. Brewe and D. Dowson, Simplified solution for elliptical-contact deformation between two elastic solids, J Lubrication Technology 99 (1977), 485-487.

[46] B.J. Hamrock and D. Dowson, Isothermal elastohydrdynamic lubrication of point contacts. Part I: theoretical formulation, J. Lubrication Technology 98 (1976), 223-229.

[47] B.J. Hamrock, Fundamental of fluid film lubrication, McGraw-Hill, NY (1984).

[48] J.A. Wensing, On the dynamic of ball bearing, Ph.D. thesis, University of Twente (1997).

[49] O.G. Gustafsson, T. Tallian, C. Barber, R. Finkelston, R. Hofman, J. Huang, R. Huston, W. Mah, J. McCool, U. Rimrott and G. Sebok, Study of the vibration characteristics of bearings, SKF Report AL63L023 (6 December 1963).

[50] M. Tiwari, K. Gupta and O. Prakash, Dynamic response of an unbalanced rotor supported on ball bearings, Journal of Sound and Vibration 238(5) (2000), 757-779.

[51] R. Aini, Vibration monitoring and modelling of shaft/bearing assemblies under concentrated elastohydrodynamic condition, Ph.D. Thesis, Kingston Polytechnic (1990).

[52] S. Fukata, E.H. Gad, T. Kondu, T. Ayabe and H. Tamura, On the radial vibrations of ball bearings (computer simulation) Bulletien of the JSME 28 (1985), 899-904.

[53] MATLAB, 2007, http://www.mathworks.com.au. 

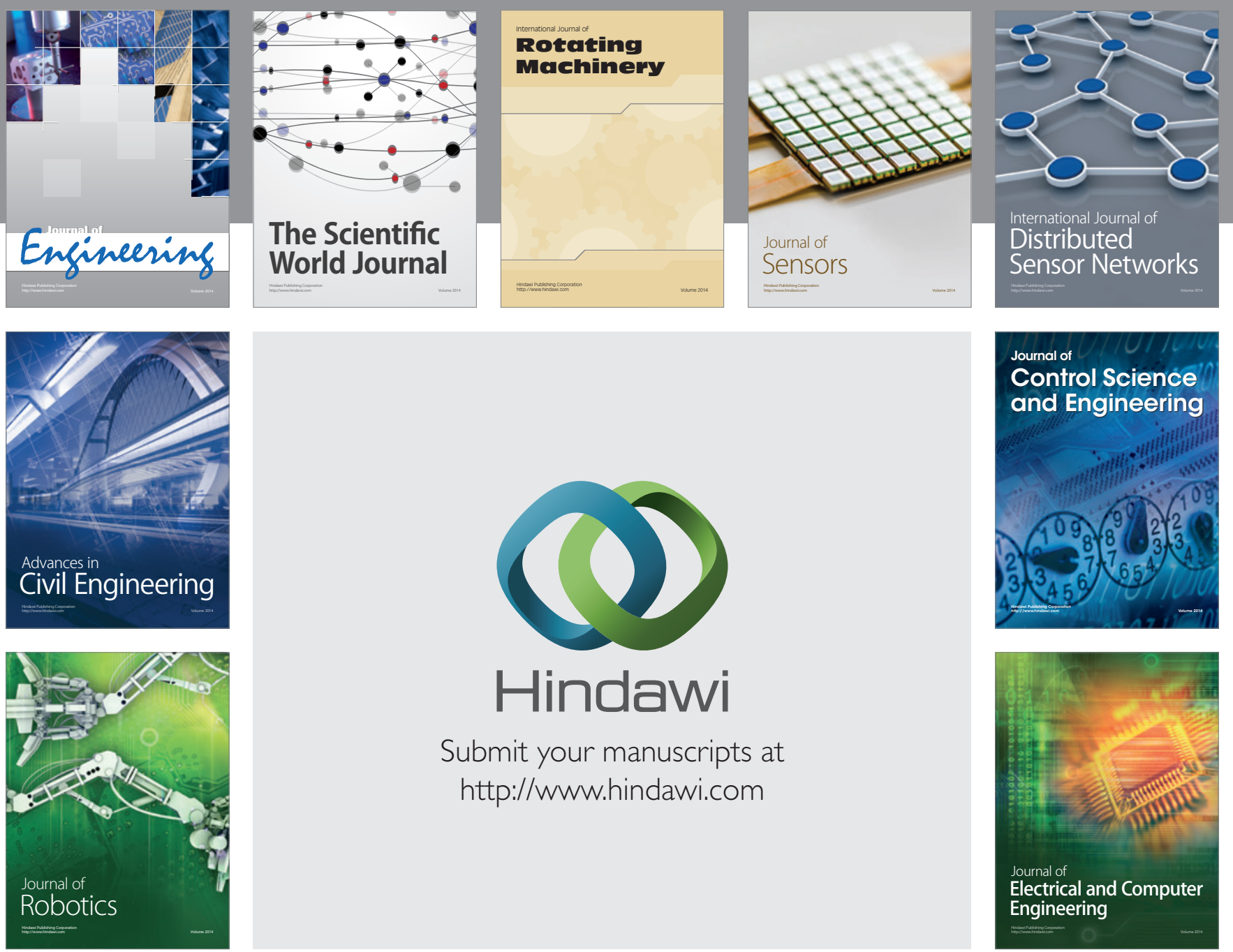

Submit your manuscripts at

http://www.hindawi.com
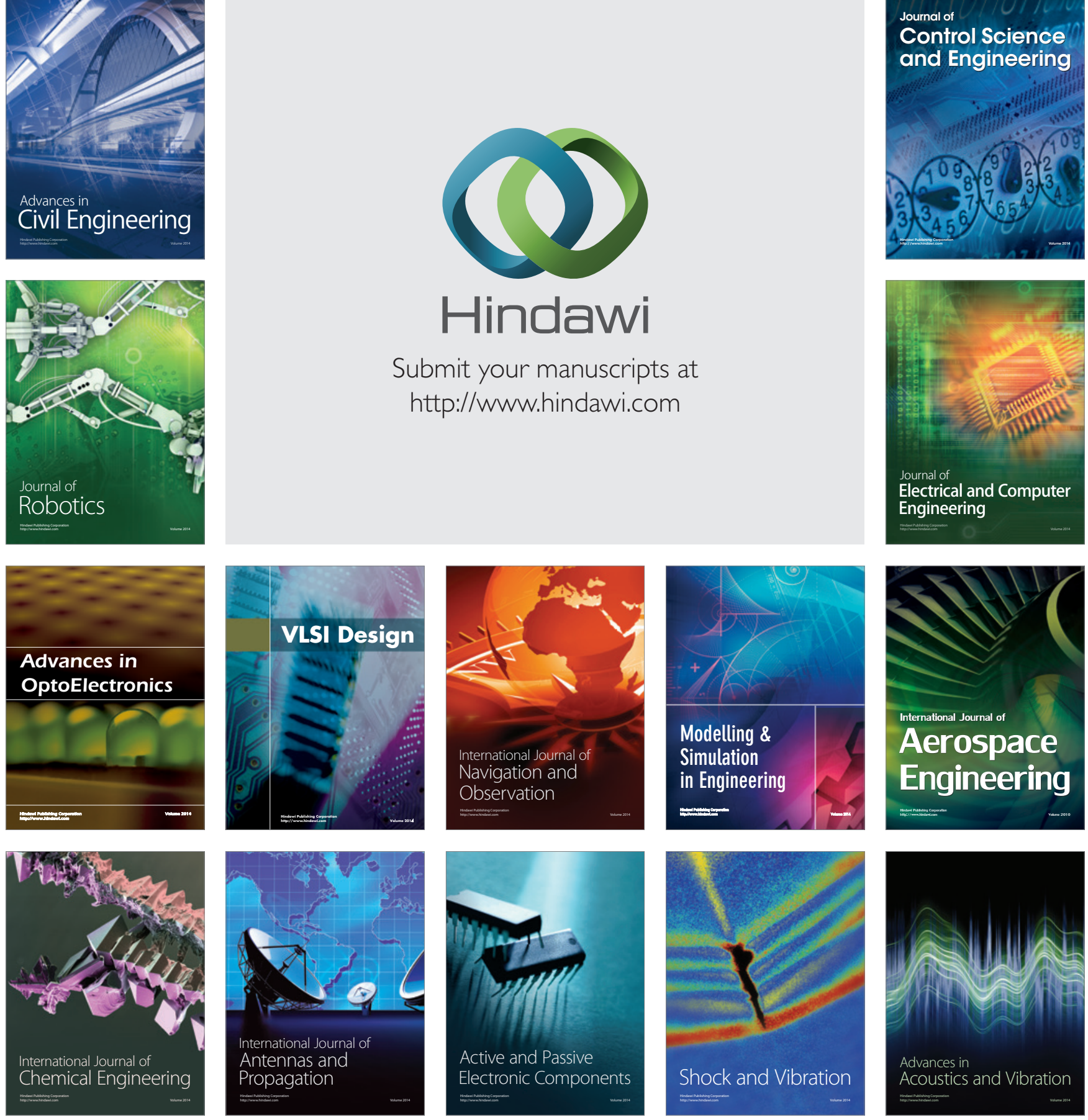